\title{
Comparing the Ca II H and K Emission Lines in Red Giant Stars
}

\author{
Jenna Ryon ${ }^{1}$ \\ Harvey Mudd College, Dept. of Physics, Claremont, CA 91711 \\ Matthew D. Shetrone \\ University of Texas, McDonald Observatory, HC75 Box 1337-McD Fort Davis, TX, 79734 \\ Graeme H. Smith \\ UCO/Lick Observatory, University of California, Santa Cruz CA 95064
}

\begin{abstract}
Measurements of the asymmetry of the emission peaks in the core of the Ca II $\mathrm{H}$ line for 105 giant stars are reported. The asymmetry is quantified with the parameter $V / R$, defined as the ratio between the maximum number of counts in the blueward peak and the redward peak of the emission profile. The Ca II $\mathrm{H}$ and $\mathrm{K}$ emission lines probe the differential motion of certain chromospheric layers in the stellar atmosphere. Data on $V / R$ for the Ca II K line are drawn from previous papers and compared to the analogous $\mathrm{H}$ line ratio, the $\mathrm{H}$ and $\mathrm{K}$ spectra being from the same sets of observations. It is found that the $\mathrm{H}$ line $(V / R)_{H}$ value is +0.04 larger, on average, than the equivalent $\mathrm{K}$ line ratio, however, the difference varies with $B-V$ color. Red giants cooler than $B-V=1.2$ are more likely to have $(V / R)_{H}>(V / R)_{K}$, whereas the opposite is true for giants hotter than $B-V=1.2$. The differences between the Ca II $\mathrm{H}$ and $\mathrm{K}$ line asymmetries could be caused by the layers of chromospheric material from which these emission features arise moving with different velocities in an expanding outflow.
\end{abstract}

\section{Introduction}

The chromospheric emission component of the Ca II K line has been studied extensively in the optical spectra of giant stars (e.g., Wilson 1976, Blanco et al. 1976, Stencel 1978, Kelch et al. 1978, Gray 1980, Middelkoop \& Zwaan 1981, Middelkoop 1982, Rutten 1984).

\footnotetext{
${ }^{1}$ REU student at McDonald Observatory
} 
The Ca II H line has not been used as often to probe the stellar atmosphere, though both lines exhibit emission in their cores that typically has a double-peaked profile. In the $\mathrm{H}$ line, the violet- and red-side emission peaks can be labeled $\mathrm{H}_{2 V}$ and $\mathrm{H}_{2 R}$ respectively. A reabsorption feature denoted $\mathrm{H}_{3}$ is located at the core of the emission profile, and is produced higher in the chromosphere. The $\mathrm{H}_{2 V}$ and $\mathrm{H}_{2 R}$ peaks often do not have equal strength. To quantify this asymmetry, it is convenient to use the $V / R$ parameter of Wilson (1976) and Stencel (1978), which we define as the ratio of intensities in the $\mathrm{H}_{2 V}$ and $\mathrm{H}_{2 R}$ emission peaks. Asymmetric emission profiles with $V / R$ values less than unity can be interpreted as evidence for an outflow having a differential velocity field in the chromospheric region, with the $\mathrm{H}_{3}$ absorption feature being blueshifted with respect to the emission profile (Stencel 1978). It is possible that the $\mathrm{Ca}$ II $\mathrm{H}$ line probes a different level of the stellar chromosphere than the $\mathrm{K}$ line, depending upon the mechanism for setting the relative populations in the upper states of these transitions (e.g., Avrett 1966; Linsky 1970). With the greater opacity of the $\mathrm{K}$ line leading to formation at higher altitudes, these emission lines can potentially be used to probe the onset of mass outflows within two different layers of the chromosphere.

In this paper we report the results from a spectroscopic program to measure the $V / R$ parameter of the Ca II H line for a sample of red giant stars. The asymmetry of the Ca II $\mathrm{H}$ emission profile is compared to that of the $\mathrm{K}$ line. Our study uses spectra of 105 red giants observed during a ten-year period, and includes 98 stars previously investigated by Smith \& Shetrone $(2000,2004)$ and Shetrone et al. (2008). The sample covers a range of stellar colors and luminosities in an effort to map out the behavior of the $\mathrm{H}$ emission as a function of location in the H-R diagram. In addition, multiple observations were obtained for a number of stars. Variability of the $\mathrm{K}$ emission line profile based on our data set has been discussed by Shetrone et al. (2008). Although time variability is not the intended subject of this paper, the individual observations listed in Table 1 allow an interested reader to judge the degree to which the $\mathrm{H}$ line asymmetry can vary on a timescale of months.

\section{Observations and Reduction}

High resolution spectra of the Ca II H and K lines for a sample of 105 field giants were obtained at McDonald Observatory. The spectra were acquired during several observing runs between October 1998 and June 2008 using either the Sandiford Cassegrain Echelle (CE) spectrometer on the 2.1-m Otto Struve Telescope or the 2d-coudé spectrometer on the 2.7-m Harlan J. Smith Telescope. For the 2.7-m we used a 1.2" slit which yielded a 2.0 pixel resolution of $R=60,000$. For the 2.1-m we used a 1.1" slit which yielded a 2.0 pixel resolution of $R=60,000$. This paper examines the Ca II H line found in a different order 
of the same echelle spectra for which K line observations have been reported upon by Smith \& Shetrone $(2000,2004)$ and Shetrone et al. (2008). To this material we add more recent $\mathrm{H}$ and $\mathrm{K}$ line data from the spectra obtained in June 2008.

The observing procedure and data reduction process for the spectra obtained during observing runs from October 1998 through August 2007 are described in Smith \& Shetrone (2000, 2004) and Shetrone et al. (2008). The most recent data were taken during the nights of 2008 June 22 and 23 using the 2d-coudé spectrometer on the 2.7-m telescope. For this later run, the observing procedure was to take at least three exposures of each object with the maximum exposure time being $1200 \mathrm{~s}$. Bias frames and flat field frames were taken at the end of each night. In the case of the 2.1-m program, since the spectrometer is a cassegrain instrument, exposures of ThAr arcs were made before and after observation of each star. By contrast, the 2d-coudé spectrometer of the 2.7-m telescope is very stable, and for the observing runs with this instrument, ThAr arcs were generally obtained only at the start and end of each night. The spectra were bias corrected, flat-field corrected, and wavelength calibrated using routines within the IRAF echelle package. The individual spectra for each object taken on a given night were combined to remove cosmic rays. During the data reduction process, the number of observed electrons was preserved to keep the Poisson statistics valid for all coadded spectra.

Using the IRAF fxcor and dopcor packages, the spectra were shifted to the velocity frame of the Hinkle spectral Atlas of Arcturus (Hinkle et al. 2000). The spectra were then divided by the Arcturus Atlas to obtain a continuum residual, which was fit by a low order Legendre polynomial, excluding the core of the $\mathrm{Ca}$ II $\mathrm{H}$ line in the fitted sample. The combined spectra were divided by the continuum fit to obtain normalized spectra. This normalization procedure matches the procedures found in Smith \& Shetrone (2004) and Shetrone et al. (2008). The original spectrum, before continuum normalization, was also saved and referenced to determine the Poisson noise.

\section{Results and Discussion}

\subsection{The Asymmetry of the Ca II $\mathrm{H}_{2}$ Emission}

Our sample is comprised of 105 giants that show double-peaked Ca II H and K emission lines in their spectra. The stars observed are listed in Table 1 along with each date on which they were observed and the $(V / R)_{H}$ value for the $\mathrm{H}$ emission feature. The error (denoted

$\left.\epsilon_{H}\right)$ on each $(V / R)_{H}$ value is calculated from the relation $\epsilon_{H}^{2}=\sigma_{V}^{2} / I_{H 2 R}^{2}+I_{H 2 V}^{2} \sigma_{R}^{2} / I_{H 2 R}^{4}$, as described in Shetrone et al. (2008). In this equation, the recorded photon counts in the $\mathrm{H}_{2 V}$ 
and $\mathrm{H}_{2 R}$ peaks of the unnormalized spectra are represented by $I_{H 2 V}$ and $I_{H 2 R}$, respectively. Taking the square root of $I_{H 2 V}$ and $I_{H 2 R}$ gives the Poisson errors in these counts, denoted $\sigma_{V}$ and $\sigma_{R}$, respectively.

The possible effects of using a different template spectrum other than the Arcturus atlas for the normalization procedure were explored using synthetic spectra. Model red giant spectra (normalized to their continua) were computed for effective temperatures of $T_{\text {eff }}=3800,4130,4540$, and $5250 \mathrm{~K}$, which span the range in temperature encompassed by the stars in our sample. Mismatches in spectral type associated with using these templates in the normalization of the observed spectra were found to introduce possible systematic effects in derived $(V / R)$ values on the level of $\approx 0.005$, which is relatively small compared to uncertainties due to photon-statistics (see Table 1). For the random errors associated with the normalization process, which vary according to where the fitting points are set outside the $\mathrm{H}$ and $\mathrm{K}$ lines, the errors are typically 0.007 on $(V / R)_{K}$ and 0.003 on $(V / R)_{H}$, which are again considerably smaller than Poisson noise in the photon counts. While the exact template used for normalization is not critical, it is important that the normalization be done. A test of the $(V / R)$ values in the unnormalized spectra revealed differences of -0.02 to -0.05 for the $(V / R)_{H}$ depending upon the color of the star.

As noted, we have simultaneous spectra of the Ca II $\mathrm{K}$ emission line for each $\mathrm{H}$-line observation listed in Table 1, these two features falling in different echelle orders of the same CCD exposure. The $(V / R)_{K}$ value of the Ca II K emission feature for each observation, and its error $\epsilon_{K}$, are also reported in Table 1. These values were taken from Smith \& Shetrone (2004) and Shetrone et al. (2008), with the exception of the June 2008 measurements. Several $(V / R)_{K}$ values were taken from Smith \& Shetrone (2000) and adjusted with a zero point offset of +0.026 to correct for differences between normalization procedures, as described in Shetrone et al. (2008). The Smith \& Shetrone (2000, 2004) papers did not include errors in the $(V / R)_{K}$ data. To estimate these we first determined an average difference between the errors in $(V / R)_{H}$ and $(V / R)_{K}$ from the Shetrone et al. (2008) data and the $\mathrm{H}$ analysis done here. This average difference, $\epsilon_{H}-\epsilon_{K}$, is 0.005. This offset was applied to the $\epsilon_{H}$ values derived from our analysis of the older Ca II H line spectra to estimate corresponding values of $\epsilon_{K}$. These new $\epsilon_{K}$ error estimates, the $\epsilon_{K}$ errors from Shetrone et al. (2008), and the $\epsilon_{H}$ errors from this analysis are listed in Table 1.

Photometry in the Johnson $B V$ system was taken from the General Catalogue of Photometric Data (GCPD; Mermilliod et al. 1997), accessed from an online catalog2. The $B-V$ color of each star, as well as the absolute visual magnitude derived from Hipparcos parallaxes

\footnotetext{
${ }^{2}$ Available at http://obswww.unige.ch/gcpd/gcpd.html.
} 
(assuming zero interstellar absorption), are listed in Table 2. The parallaxes were tabulated in the earlier papers of Smith \& Shetrone $(2002,2004)$. The distance range among most of the stars in our sample is 20-200 pc, with 9 stars of known parallax being beyond 200 pc. Also listed in Table 2 is the heliocentric radial velocity $v_{r}$ as obtained from the SIMBAD database and the metallicity from McWilliam (1990) of each star. These properties are all consistent with our sample being Population I giants.

In Figure 1 the asymmetry $(V / R)_{H}$ of the Ca II $\mathrm{H}$ line is plotted versus the $B-V$ color of each star. In cases of stars observed multiple times, each observation is plotted separately, and consequently this diagram contains the effects of any time variability in the $\mathrm{H}$ line asymmetry. With the exception of one observation of HD 203387, only stars with $B-V>1.2$ exhibit red-dominant asymmetry where $(V / R)_{H}<1.0$. The most extreme red asymmetries where $(V / R)_{H}<0.7$ are only seen in stars with $B-V>1.5$. Figure 1 can be compared to Figure 2 from Shetrone et al. (2008), which gives an analogous plot for the asymmetry parameter of the Ca II K line. Both plots illustrate two groupings of data points spanning similar asymmetry and color ranges: one in the region $1.0<(V / R)<1.3$ and $0.85<(B-V)<1.05$, and the other with $0.7<(V / R)<1.4$ and $1.2<(B-V)<1.45$. However, data for giants redder than $B-V=1.45$ are unique to Figure 1, which represents a more diverse sample of stars than that in Shetrone et al. (2008).

\subsection{The Issue of Interstellar Absorption}

Stellar $\mathrm{H}$ and $\mathrm{K}$ emission line profiles can be affected by Ca II absorption in the interstellar medium (ISM). Although we do not have spectra of the interstellar Ca II lines in the directions of our program stars, several types of evidence indicate that the asymmetry trends seen in the $(V / R)$ data are not attributable to systematic interstellar corruption of the stellar Ca II lines.

If interstellar absorption was dominating the observed $(V / R)$ values then the sense of the asymmetry should correlate with the velocity of each star relative to the ISM (as in Figure 5 of Böhm-Vitense 1981 for the Mg II $k_{2}$ emission). Several diagrams were prepared to test whether a similar correlation is present in the Ca II data. Plots were made of the average $(V / R)_{K}$ and average $(V / R)_{H}$ values of each star versus heliocentric radial velocity $v_{r}$; no trend is seen (Figure 2). Furthermore, a version of Figure 1 was made using different symbols to depict stars in different radial velocity ranges: $v_{r}<-20 \mathrm{~km} \mathrm{~s}^{-1},-20<v_{r}<+20$

$\mathrm{km} \mathrm{s}^{-1}$, and $v_{r}>20 \mathrm{~km} \mathrm{~s}^{-1}$. There is no evidence that stars with positive or negative $v_{r}$ occupy systematically different regions of the diagram. We conclude that the Ca II emission lines are more resilient to ISM corruption than found by Böhm-Vitense (1981) for the Mg II 
$h$ and $k$ emission.

As a second test, the ISM absorption $A_{V}$ towards most program stars was calculated using the numerical recipe of Hakkila et al. (1997), which takes into account both the distance to each star and the Galactocentric coordinates of each star. Many of the stars from Table 1 are within $75 \mathrm{pc}$ of the Sun, and within a local bubble inside of which ISM extinction is considered to be small or negligible (e.g., Sfeir et al. 1999; Luck \& Heiter 2007). The equivalent width of the interstellar Ca II K line as seen against early-type stars within $100 \mathrm{pc}$ of the Sun tends to be less than $10 \mathrm{~m} \AA$ (e.g., Vallerga et al. 1993), although exceptions occur. The values of $A_{V}$ derived according to the Hakkila et al. (1997) model, modified such that $A_{V}$ is set to zero within $75 \mathrm{pc}$, are listed in Table 2. For the majority of program stars, the estimated $A_{V}<0.1$ mag. Furthermore, those stars with the largest values of $A_{V}$ do not occupy systematically discordant positions in the $\mathrm{H}$ and $\mathrm{K}$ line $(V / R)$ versus $(B-V)$ diagrams. In summary, considering both the radial velocities of the program stars, and the estimated interstellar extinction towards them, we see no systematics of the type that would be expected if ISM corruption of the Ca II H and $\mathrm{K}$ emission profiles was governing the observed $(V / R)$ values.

\subsection{Differences Between the $\mathrm{H}$ and $\mathrm{K}$ Line Asymmetries}

Despite the similarities between Figure 1 of this paper and Figure 2 of Shetrone et al. (2008), there are nonetheless differences between $(V / R)_{H}$ and $(V / R)_{K}$ for many stars. Some illustrative examples of asymmetry differences are shown in Figures 3 and 4 . The first of these shows spectra of HD 156283 obtained on 22 June 2008 using the 2.7-m telescope, wherein the $(V / R)_{K}$ ratio is 0.99 and $(V / R)_{H}$ is 1.07. At this time, although the $\mathrm{K}$ emission line was nearly symmetrical, the $\mathrm{H}$ line showed a blue-enhanced asymmetry in which the violet $\mathrm{H}_{2 V}$ peak was stronger than the red peak. Figure 4 shows a spectrum of HD 21552 obtained on 3 October 2001 using the 2.1-m telescope. The Ca II $(V / R)_{K}$ ratio for this spectrum is 0.94 while $(V / R)_{H}=1.01$. In this case, although the $\mathrm{H}$ line is nearly symmetric, the $\mathrm{K}$ emission profile is stronger in the red peak.

The difference between the $\mathrm{Ca}$ II $\mathrm{H}$ and $\mathrm{K}$ line $V / R$ values was calculated for every observation of each star in the sample. The resulting set of difference values is plotted versus the $\mathrm{H}$ line $(V / R)_{H}$ in Figure 5; each observation of each star is plotted, so some stars are represented by multiple points. The error bars were calculated by summing $\epsilon_{H}$ and $\epsilon_{K}$ in quadrature. The value of $(V / R)_{H}$ is larger than $(V / R)_{K}$ by an average offset of +0.039 , which is shown as a dashed line in Figure 5. However, it can be seen that there are trends within this diagram, such that a single average offset is not a good fit to the entire data set. 
The difference between the $\mathrm{H}$ and $\mathrm{K}$ line $V / R$ values are shown in Figure 6 versus $B-V$ color. As with Figure 5, asymmetry values from individual spectra are plotted, so that some stars are depicted with multiple data points. The linear best fit to the data is plotted as a dashed line, which has a slope of $0.162 \pm 0.017$. However, this fit does not seem to be the best representation of the data. In order to smooth over possible intrinsic variability, an average of the $(V / R)_{H}-(V / R)_{K}$ values was calculated for each star. These averages are shown plotted versus $B-V$ in Figure 7 , so that unlike Figure 6, each star is depicted by just one data point. In order to further smooth the data to look for trends, a weighted mean was made of every five points in Figure 7 binned according to $B-V$. A continuous line shows the locus of these binned averages. Rather than a linear relation, these binned data exhibit a quasi-step function with a reasonably abrupt change at $(B-V) \sim 1.25$. The two horizontal lines in the figure show the average value of the asymmetry difference among giants with colors on either side of this "divide."

The color-magnitude diagram for our sample of red giants is plotted in Figure 8. The data points are coded according to the significance level of the $(V / R)_{H}-(V / R)_{K}$ values; open circles and open squares represent stars for which the average asymmetries differences are $\left\langle(V / R)_{H}-(V / R)_{K}\right\rangle>1 \sigma$ and $\left\langle(V / R)_{H}-(V / R)_{K}\right\rangle<1 \sigma$, respectively, while filled triangles represent stars for which $\left|\left\langle(V / R)_{H}-(V / R)_{\mathrm{K}}\right\rangle\right|<1 \sigma$. Red giants for which $(V / R)$ is notably greater in the $\mathrm{H}$ line than the $\mathrm{K}$ line (open circles) tend to have colors of $(B-V)>1.2$, while stars for which the reverse is the case (open boxes) are mainly of $(B-V)<1.2$, although they do extend as red as $(B-V)=1.4$. Giants with similar $\mathrm{H}$ and $\mathrm{K}$ line asymmetries (filled triangles) are evenly spread across the entire $B-V$ range covered by the data sample. Thus, stars with similar asymmetry differences tend to group in specific regions of the color-magnitude diagram, albeit with some overlap, particularly in the color range $1.2<(B-V)<1.4$. This is a further reflection of the trend seen in Figure 6 .

\subsection{Photospheric Effects}

The Ca II H and K line chromospheric emission components are superimposed on a photospheric absorption spectrum that varies with both wavelength and effective temperature. Could the differences between $(V / R)_{\mathrm{H}}$ and $(V / R)_{\mathrm{K}}$ be due to differences in the background photospheric spectrum in the vicinity of the $\mathrm{H}$ and $\mathrm{K}$ lines? We have investigated this question through the use of synthetic photospheric spectra calculated using the 2007 version of the program MOOG (Sneden 1973). These spectra were generated for a model atmosphere

with $T_{\text {eff }}=4250 \mathrm{~K}, \log g=1.75$, and $[\mathrm{Fe} / \mathrm{H}]=-0.1$, a combination of parameters that is typical of the red giants in our sample. Synthetic photospheric spectra were generated for 
three linelists: one with all of the photosphere lines, a second list from which the $\mathrm{H}$ and $\mathrm{K}$ lines had been removed, and a third that contained only the $\mathrm{Ca} \mathrm{II} \mathrm{H}$ and $\mathrm{K}$ lines. To simulate a symmetric double-peaked $\mathrm{H}$ or $\mathrm{K}$ emission feature, two equal-height Gaussian profiles were combined having separations in wavelength that are typical of the observed spectra. In accordance with the optically thin case, the relative strengths of these $\mathrm{H}$ and $\mathrm{K}$ emission profiles were set to be 1:2, with the peak flux in the $\mathrm{K}$ emission being $15 \%$ that of the continuum level of the photospheric spectrum on which it is superimposed. Mathematically, the flux of these artificial peaks was simply added to the synthetic photospheric spectrum computed with the full line list.

A decomposition of the resultant model spectrum is presented in the various panels of Figure 9, which show from top to bottom: the $\mathrm{H}$ and $\mathrm{K}$ photospheric lines alone, all photospheric lines other than $\mathrm{H}$ and $\mathrm{K}$, the complete photospheric spectrum, the artificial $\mathrm{H}$ and $\mathrm{K}$ emission profiles, and the full photospheric spectrum plus the artificial emission lines. There are numerous weak and moderate strength absorption lines throughout the wavelength range $3925<\lambda<3980 \AA$, but once they get superimposed on the very broad and deep photospheric profiles of the $\mathrm{H}$ and $\mathrm{K}$ lines, the amplitudes of these weaker lines are greatly reduced. Since the total photospheric flux in the cores of these lines is relatively small, the effect of the photospheric background on the chromospheric emission profiles is very modest. In Figure 9, the asymmetry induced by this effect is barely discernible.

To complement the simulation, photospheric models were also computed for a range of effective temperatures. As with Figure 9, simulated $\mathrm{H}$ and $\mathrm{K}$ emission lines, both with $(V / R)=1$ and a $\mathrm{K}$ line twice as strong as the $\mathrm{H}$ line, were added to the set of synthetic photosphere spectra. From these superimposed spectra the values of $(V / R)_{K},(V / R)_{H}$, and $(V / R)_{H}-(V / R)_{K}$ were calculated. In addition to simulations with symmetric emission profiles, calculations were also done for input values of $(V / R)_{H, K}=1.2$ and 0.6 , as well as a range of wavelength separations between the two maxima in each bimodal profile. In all cases the input $\mathrm{H}$ and $\mathrm{K}$ emission profiles had identical asymmetry, such that initially $(V / R)_{H}-(V / R)_{K}=0$. The value of this difference after superimposing the model photosphere spectrum illustrates the extent to which photospheric corruption might be affecting the observed asymmetry differences. The resultant values of $(V / R)_{H}-(V / R)_{K}$ are plotted versus $B-V$ in Figure 10. Modest systematic variations are seen as a function of $(B-V)$, which are largely driven by photospheric corruption of the $\mathrm{K}$ line rather than the $\mathrm{H}$ line. However, the magnitude of these asymmetry differences is much smaller (by a factor of 5) than the star-to-star differences, and their trend with $(B-V)$, than we actually observe. Although this modeling approach is no substitute for a self-consistent treatment of radiative transfer through a combined model photosphere plus chromosphere, it does suggest that the $(V / R)$ asymmetry variations among red giants are not an artifact of photospheric differences, 
but reflect physical differences in the stellar chromospheres.

In computing the synthetic spectra it was assumed that there is no systematic velocity shift between the simulated emission profiles and the photospheric spectrum. As noted above, in the data reduction procedure all of the observed spectra were shifted so that the photospheric lines are at the rest wavelengths as defined by the Arcturus atlas. The average central wavelengths of the $\mathrm{H}$ and $\mathrm{K}$ emission profiles were measured in this rest frame for the 8 stars observed at the $2.7-\mathrm{m}$ telescope in 2008. There was no systematic displacement of the emission line centers from the photospheric reference frame. The average deviation was found to be $-0.02 \mathrm{~km} \mathrm{~s}^{-1}$ with a standard deviation of $1.0 \mathrm{~km} \mathrm{~s}^{-1}$ (1 pixel $=2.4 \mathrm{~km}$ $\left.\mathrm{s}^{-1}\right)$, and there is no trend with $(B-V)$ color.

\section{Conclusions}

Values of the asymmetry parameter $(V / R)_{H}$ for the $\mathrm{Ca}$ II $\mathrm{H}$ chromospheric emission line have been measured for a large sample of red giant stars. The results are presented in Table 1, plotted as a function of $B-V$ in Figure 1, and in a series of other diagrams are compared to $V / R$ values of the Ca II K line based on the same spectroscopic data set (see Shetrone et al. 2008). Many of the spectra exhibit opposite asymmetries between the $\mathrm{H}$ and $\mathrm{K}$ line emission peaks. The $\mathrm{H}$ line $(V / R)_{H}$ value is larger than the $(V / R)_{K}$ ratio for the majority of stars in our sample, however, this difference shows a trend with $B-V$ color, being largest among giants with $(B-V)>1.25$.

The observations can be interpreted within a framework in which the $\mathrm{H}$ and $\mathrm{K}$ emission lines are formed at different levels in a stellar chromosphere, thereby allowing for a comparison between the motions of these layers. Asymmetry in either the $\mathrm{H}$ or $\mathrm{K}$ line, i.e., $V / R$ different from unity, is generally attributed to Doppler shifts resulting from a differential velocity field in the chromosphere. In this Doppler picture, gas that produces the $\mathrm{H}_{2 V}$ and $\mathrm{H}_{2 R}$ peaks is taken to be moving with a different velocity to the gas that contributes to the central $\mathrm{H}_{3}$ reversal. This causes the $\mathrm{H}_{3}$ reversal to be redshifted or blueshifted with respect to the $\mathrm{H}_{2}$ peaks, resulting in an emission line asymmetry. Such a scenario has been invoked, for example, to interpret $\mathrm{H}$ and $\mathrm{K}$ line asymmetry differences among dwarf stars (Rauscher \& Marcy 2006).

On the Sun the $V / R$ values for the $\mathrm{H}$ emission line follow a slightly skewed distribution with a maximum at unity, a minimum of $(V / R)_{H} \approx 0.5$, and an extended tail to values greater than 1.7 (Cram \& Damé 1983). There is only a weak correlation between $(V / R)_{\mathrm{H}}$ and the strength of the $\mathrm{H}$ emission, from which Cram \& Damé infer that "the $V / R$ ratio 
of the integrated solar $\mathrm{H}$ line is not due specifically to bright (e.g., network) elements". Furthermore, the $\mathrm{H}_{3}$ reversal is found to be consistently displaced away from whichever of the $\mathrm{H}_{2 V}$ and $\mathrm{H}_{2 R}$ peaks is brightest, which indicates that Doppler velocity shifts of the $\mathrm{H}_{3}$ reversal are an important factor governing the asymmetry of the $\mathrm{H}$ emission profile (Cram \& Damé 1983). Enhanced emission in the $\mathrm{H}_{2 V}$ peak appears to be associated with shock heating (e.g., Beck et al. 2008). In regions of the quiet Sun, the $\mathrm{H}_{2 V}$ and $\mathrm{H}_{2 R}$ emission have even been observed to disappear, indicative of cool elements within cells of the chromospheric network (Rezaei et al. 2008).

Among the majority of dwarf stars with spectral types in the range K7-M5, Rauscher \& Marcy (2006) show that $V / R>1$ in both the $\mathrm{H}$ and $\mathrm{K}$ lines, with a preference for $(V / R)_{K}>$ $(V / R)_{H}$. In addition, the $\mathrm{K}_{3}$ reversal tends to have a systematically greater redshift than the $\mathrm{H}_{3}$ feature. It is worth quoting the conclusions of Rauscher \& Marcy on this point: "While the unequal degeneracies in the upper levels producing these lines cause somewhat differing optical depths, these data emphasize that the $\mathrm{H}$ and $\mathrm{K}$ lines are produced in slightly different regions of the chromosphere and are therefore governed by slightly different line transfer and kinematics." The redshift of the central reversals is attributed by them to micro-flare activity that heats chromospheric gas and propels it outwards. As it moves upward it cools and decelerates, resulting in the gas that contributes to the central reversal being redshifted relative to the faster moving, hotter gas below it that produces the emission peaks. The velocity differential between these regions of gas is on the order of several tenths of a $\mathrm{km}$ $\mathrm{s}^{-1}$. The greater redshift of the $\mathrm{K}_{3}$ reversal compared to the $\mathrm{H}_{3}$ reversal is consistent with the former arising from slightly higher altitudes in the chromosphere, where deceleration has proceeded to a greater degree.

Clues to the systematics of the chromospheric motions at work within our sample of red giants can be found in Figures 11 and 12. Average values of $(V / R)_{H}$ and $(V / R)_{K}$ were calculated for each star using the data in Table 1. The main panel of Figure 11 shows these average asymmetries plotted versus one another. In Figure 12 both $\left\langle(V / R)_{H}\right\rangle$ and $\left\langle(V / R)_{K}\right\rangle$ are plotted versus the average value of the asymmetry difference $\left\langle(V / R)_{H}-(V / R)_{K}\right\rangle$. This diagram is analogous to Figure 5, except that all data for a given star are averaged, so that each giant is represented by a single point. These figures map out the relative behavior of the $\mathrm{H}$ and $\mathrm{K}$ emission line profiles.

Among giants with the largest values of $(V / R)_{H}(>1.1)$ this asymmetry parameter is nearly equal in value to $(V / R)_{K}$; such stars exhibit a blue-dominant asymmetry in both the $\mathrm{H}$ and $\mathrm{K}$ emission profiles, and scatter closely around the locus $(V / R)_{H}=(V / R)_{K}$ which is shown by the straight line in Figure 11. Among such stars it seems that the $\mathrm{H}$ and $\mathrm{K}$ lines are evincing similar velocity fields and that there is no evidence of systematic outflow in the 
chromosphere.

Turning to giants with $1.0<(V / R)_{H}<1.1$, there is a noticeably larger range of $\mathrm{K}$ line asymmetries and one starts to see a systematic shift of the $\mathrm{K}$ line profiles to reddominant asymmetries, i.e., to $(V / R)_{K}<1.0$. A significant fraction of giants with $1.0<$ $(V / R)_{H}<1.1$ also have $(V / R)_{K}<1.0$, and so exhibit opposite $\mathrm{H}$ and $\mathrm{K}$ emission profile asymmetries. Among such stars we infer that an accelerating outflow has become established within a region of the chromosphere that spans altitudes at which the $K_{2}$ and $K_{3}$ features are formed. We interpret the later feature as being formed at higher levels, in regions of faster outflow, than $\mathrm{K}_{2}$. This causes the central absorption reversal to be blueshifted with respect to the emission profile, thereby giving a characteristic asymmetry of $(V / R)_{K}<1.0$. However, the outflow does not yet extend to the deeper altitudes where the $\mathrm{H}_{3}$ feature is formed. It is therefore a change in the $\mathrm{K}$ line asymmetry that drives the non-zero values of $\left\langle(V / R)_{H}-(V / R)_{K}\right\rangle$ among giants having $1.0<(V / R)_{H}<1.1$. As a result, many of these stars fall below the straight line in Figure 11. This phenomenon is reflected by a plateau of approximately constant $(V / R)_{H} \sim 1.1 \pm 0.05$ in the upper panel of Figure 12, whereas the same stars fall along an inclined locus in the lower panel.

Eventually the accelerating outflow extends downwards in altitude to regions where the $\mathrm{H}_{3}$ feature is formed, resulting in $(V / R)_{H}$ now decreasing to a value below unity. This brings about a maximum in the mean difference $\left\langle(V / R)_{H}-(V / R)_{K}\right\rangle$ of $\sim 0.2$. Both $(V / R)_{H}$ and $(V / R)_{K}$ are now less than unity and we infer that an accelerating wind now extends throughout the chromospheric regions where the $\mathrm{K}_{3}, \mathrm{~K}_{2}, \mathrm{H}_{3}$, and possibly the $\mathrm{H}_{2}$ features originate. As the value of $(V / R)_{H}$ drops below 0.8 the difference in $V / R$ between the $\mathrm{H}$ and $\mathrm{K}$ lines diminishes, and for stars with $(V / R)_{H} \sim 0.4-0.5$ the asymmetry parameters for the two lines become approximately equal. Such giants may be those with the largest velocity gradients in the chromosphere. They have returned to the vicinity of the straight line plotted in Figure 11.

Figure 1 shows that the systematic trends outlined in the previous paragraphs correspond approximately to a sequence in $B-V$ color. Thus it may be that as stars evolve up the red giant branch they travel along an evolutionary sequence in Figures 11 and 12, with winds first originating at high altitudes in the chromosphere, and then becoming more established and extending to lower altitudes.

However, it is also known that $V / R$ for the $\mathrm{H}$ and $\mathrm{K}$ lines can be time variable (Shetrone et al. 2008), and this is likely to impose scatter on any long-term evolutionary sequence. An excellent example is the star Arcturus, which both Gray (1980) and Qin \& Li (1987) find to be variable over timescales of hours to months. This indicates that red giant atmospheres are likely subject to both short and long term dynamical modification. Changes on a timescale 
of hours were interpreted by Qin \& Li (1987) as being due to a "chromospheric eruption" that may have ejected mass rapidly through the chromosphere and possibly to escape from the star.

In the solar chromosphere significant differences in the ratio of $\mathrm{H}$ and $\mathrm{K}$ line source functions can occur over a range in height of approximately 500-700 km (Linsky 1970). Comparing to a $\mathrm{G}$ giant of solar effective temperature and mass, and radius $R=40 R_{\odot}$, the chromospheric scale height might be expected to vary roughly as the inverse surface gravity, i.e., as $R^{2}$. Thus, variations in the ratio of the $\mathrm{H}$ and $\mathrm{K}$ line source functions might extend over an altitude range of $\approx 800,000 \mathrm{~km}$. A disturbance moving at $5 \mathrm{~km} \mathrm{~s}^{-1}$ could traverse such a region in about 44 hours. Although we do have multiple observations of many stars, the typical time difference between consecutive spectra is months or years rather than days. Thus, in general, we don't have fine enough temporal coverage to detect possible systematic variations in $\mathrm{H}$ or $\mathrm{K}$ line asymmetries due to passage of a short-term "event" through the chromosphere. In the case of HD 27697, the two spectra obtained in 1998 on consecutive nights reveal no evidence for any significant differences in $(V / R)$ for either the $\mathrm{H}_{2}$ or $\mathrm{K}_{2}$ features. Similarly, spectra of HD 82635 taken 18 nights apart in 2007 show no significant evidence of variability. In 2006, the data show that both the $\mathrm{H}_{2}$ and $\mathrm{K}_{2}(V / R)$ values for HD 222404 differed significantly between 1 September and 4 October, but our observations were a month, rather than several days, apart.

It should be noted, however, that the $\mathrm{H}$ and $\mathrm{K}$ line observations of Arcturus by Qin \& Li (1987) may depict a very energetic version of a short-timescale phenomenon. The asymmetry indices measured by them (using their notation where $r$ refers to the intensity in an emission line peak) vary within the range $0.87<r\left(\mathrm{H}_{2 V}\right) / r\left(\mathrm{H}_{2 r}\right)<1.21$ and $0.79<r\left(\mathrm{~K}_{2 V}\right) / r\left(\mathrm{~K}_{2 r}\right)<$ 0.93 over a period of 4 hours, and all their observations show $r\left(\mathrm{H}_{2 V}\right) / r\left(\mathrm{H}_{2 r}\right)>r\left(\mathrm{~K}_{2 V}\right) / r\left(\mathrm{~K}_{2 r}\right)$. Furthermore, in 4 of their 6 spectra $r\left(\mathrm{~K}_{2 V}\right) / r\left(\mathrm{~K}_{2 r}\right)<1.0$ while $r\left(\mathrm{H}_{2 V}\right) / r\left(\mathrm{H}_{2 r}\right)>1.0$, i.e., the $\mathrm{H}$ and $\mathrm{K}$ emission lines evince opposite asymmetries. On the basis of this example, it follows that short-term variability could indeed contribute to the star-to-star scatter in asymmetries seen in the McDonald spectra. Such effects may be superimposed on a much longer-term evolutionary sequence that produces the trends with $(B-V)$ color seen in Figures 11 and 12 , and which may be associated with the onset of pulsation and/or the driving of a large scale wind.

This project was completed as part of the McDonald Observatory REU program and was supported under NSF AST-0649128. This research has made use of the Simbad database, operated at CDS, Strasbourg, France. We would like to thank E. Luck for providing the extinction code, hakkila, used in this work. 


\section{REFERENCES}

Avrett, E. H. 1966, ApJ, 144, 59

Beck, C., Schmidt, W., Rezaei, R., \& Rammacher, W. 2008, A\&A, 479, 213

Blanco, C., Marilli, E., \& Catalano, S. 1976, A\&A, 48, 19

Böhm-Vitense, E. 1981, ApJ, 244, 504

Cram, L. E., \& Damé, L. 1983, ApJ, 272, 355

Gray, D. F. 1980, ApJ, 240, 125

Hakkila, J., Myers, J. M., Stidham, B. J., \& Hartmann, D. H. 1997, AJ, 114, 2043

Hinkle, K., Wallace, L., Valenti, J., \& Harmer, D. 2000, Visible and Near Infrared Atlas of the Arcturus Spectrum 3727-9300 Å(San Francisco, CA: ASP)

Kelch, W. L., Chang, S.-H., Furenlid, I., Linsky, J. L., Basri, G. S., Chiu, H.-Y., \& Maran, S. P. 1978, ApJ, 220, 962

Linsky, J. L. 1970, Sol. Phys., 11, 355

Luck, R. E., \& Heiter, U. 2007, AJ, 133, 2464

McWilliam, A. 1990, ApJS, 74, 1075

Mermilliod, J.-C., Mermilliod, M., \& Hauck, B. 1997, A\&AS, 124, 349

Middelkoop, F. 1982, A\&A, 113, 1

Middelkoop, F., \& Zwan, C. 1981, A\&A, 101, 26

Qin, S.-N., \& Li, J.-K. 1987, Chinese Astronomy \& Astrophysics, 11, 146

Rauscher, E., \& Marcy, G. W. 2006, PASP, 118, 617

Rezaei, R., Bruls, J. H. M. J., Schmidt, W., Beck, C., Kalkofen, W., \& Schlichenmaier, R. 2008, A\&A, 484, 503

Rutten, R. G. M. 1984, A\&A, 130, 353

Sfeir, D. M., Lallemont, R., Crifo, F., \& Welsh, B. Y. 1999, A\&A, 346, 785

Shetrone M., Rivera, F., Smith, G., \& Carveth, C. 2008, PASP, 120, 730 
Smith, G.H. \& Shetrone M.D. 2000, PASP, 112, 1320

Smith, G.H. \& Shetrone M.D. 2004, PASP, 116, 604

Sneden, C. 1973, ApJ, 184, 839

Stencel, R. E. 1978, ApJ, 223, L37

Vallerga, J. V., Vedder, P. W., Craig, N., \& Welsh, B. Y. 1993, ApJ, 411, 729

Wilson, O. C. 1976, ApJ, 205, 823 
Table 1. Individual measurements

\begin{tabular}{|c|c|c|c|c|c|}
\hline $\begin{array}{l}\text { Star } \\
(\mathrm{HD})\end{array}$ & Date & $\begin{array}{c}(V / R)_{H} \\
(\mathrm{H})\end{array}$ & $\begin{array}{c}\text { error } \\
\left(\epsilon_{H}\right)\end{array}$ & $\begin{array}{c}(V / R)_{K} \\
(\mathrm{~K})\end{array}$ & $\begin{array}{r}\text { error } \\
\left(\epsilon_{K}\right)\end{array}$ \\
\hline 1013 & 2001-10-05 & 0.79 & 0.03 & 0.70 & 0.03 \\
\hline 1227 & 1998-11-28 & 1.15 & 0.07 & 1.13 & 0.06 \\
\hline 1522 & $2006-10-03$ & 1.14 & 0.05 & 1.03 & 0.05 \\
\hline \multirow[t]{2}{*}{3627} & $1998-10-25$ & 1.16 & 0.04 & 1.10 & 0.04 \\
\hline & 2001-10-08 & 1.08 & 0.02 & 1.09 & 0.03 \\
\hline \multirow[t]{2}{*}{4128} & 2006-08-31 & 1.07 & 0.03 & 1.12 & 0.03 \\
\hline & 2006-10-04 & 1.04 & 0.02 & 1.07 & 0.03 \\
\hline 4482 & 1998-10-25 & 1.11 & 0.03 & 1.10 & 0.03 \\
\hline 4730 & $2001-10-08$ & 1.06 & 0.06 & 1.06 & 0.05 \\
\hline 5516 & 1998-11-30 & 1.10 & 0.04 & 1.22 & 0.03 \\
\hline 6953 & $2001-10-05$ & 1.14 & 0.05 & 1.08 & 0.04 \\
\hline 7318 & $1998-11-26$ & 1.16 & 0.06 & 1.14 & 0.05 \\
\hline \multirow[t]{2}{*}{9138} & 2001-10-04 & 1.03 & 0.07 & 0.92 & 0.04 \\
\hline & 2006-10-04 & 0.74 & 0.05 & 0.97 & 0.09 \\
\hline 9774 & 1998-11-29 & 1.16 & 0.06 & 1.18 & 0.06 \\
\hline 11559 & $1998-11-28$ & 1.13 & 0.05 & 1.08 & 0.04 \\
\hline 11930 & $2001-10-07$ & 1.11 & 0.05 & 1.00 & 0.04 \\
\hline 14652 & $2001-10-05$ & 0.62 & 0.05 & 0.61 & 0.05 \\
\hline 15656 & $2001-10-03$ & 0.77 & 0.04 & 0.62 & 0.04 \\
\hline 15694 & $2001-10-08$ & 1.06 & 0.05 & 1.01 & 0.04 \\
\hline 16058 & $1998-10-25$ & 0.55 & 0.03 & 0.42 & 0.02 \\
\hline 17506 & 1998-11-29 & 0.64 & 0.06 & 0.61 & 0.05 \\
\hline 19349 & $2001-10-05$ & 1.04 & 0.04 & 0.98 & 0.03 \\
\hline 19476 & $1998-10-26$ & 1.16 & 0.04 & 1.22 & 0.04 \\
\hline 20610 & $1998-11-28$ & 1.19 & 0.08 & 1.15 & 0.07 \\
\hline 20893 & 2001-10-08 & 0.91 & 0.04 & 1.11 & 0.04 \\
\hline 21051 & $1998-11-26$ & 1.13 & 0.07 & 1.18 & 0.06 \\
\hline 21552 & $2001-10-03$ & 1.01 & 0.03 & 0.94 & 0.03 \\
\hline 26076 & 1998-11-27 & 1.30 & 0.10 & 1.28 & 0.09 \\
\hline 26846 & $2001-10-03$ & 1.11 & 0.04 & 1.10 & 0.03 \\
\hline
\end{tabular}


Table 1-Continued

\begin{tabular}{|c|c|c|c|c|c|}
\hline $\begin{array}{l}\text { Star } \\
(\mathrm{HD})\end{array}$ & Date & $\begin{array}{c}(V / R)_{H} \\
(\mathrm{H})\end{array}$ & $\begin{array}{c}\text { error } \\
\left(\epsilon_{H}\right)\end{array}$ & $\begin{array}{c}(V / R)_{K} \\
(\mathrm{~K})\end{array}$ & $\begin{array}{r}\text { error } \\
\left(\epsilon_{K}\right)\end{array}$ \\
\hline 27278 & $1998-11-26$ & 1.03 & 0.05 & 1.08 & 0.05 \\
\hline 27371 & $1998-11-28$ & 1.09 & 0.03 & 1.10 & 0.03 \\
\hline \multirow[t]{2}{*}{27697} & 1998-11-29 & 1.14 & 0.08 & 1.24 & 0.07 \\
\hline & 1998-11-30 & 1.13 & 0.04 & 1.19 & 0.04 \\
\hline \multirow[t]{3}{*}{28305} & $1998-11-30$ & 1.18 & 0.04 & 1.12 & 0.03 \\
\hline & 2006-10-03 & 1.20 & 0.04 & $\cdots$ & $\ldots$ \\
\hline & 2007-02-01 & 1.14 & 0.04 & 1.02 & 0.03 \\
\hline 28307 & $1998-11-30$ & 1.07 & 0.03 & 1.13 & 0.02 \\
\hline 32357 & $1998-11-26$ & 1.05 & 0.03 & 1.12 & 0.02 \\
\hline 32887 & 2001-10-08 & 0.94 & 0.03 & 0.96 & 0.03 \\
\hline 33856 & $1998-11-30$ & 1.07 & 0.06 & 1.17 & 0.05 \\
\hline 35186 & $2001-10-07$ & 1.22 & 0.04 & 1.23 & 0.04 \\
\hline 42633 & $2001-10-08$ & 1.04 & 0.05 & 1.07 & 0.05 \\
\hline 42995 & $1998-11-27$ & 0.38 & 0.05 & 0.39 & 0.04 \\
\hline \multirow[t]{4}{*}{49161} & $1998-11-28$ & 1.08 & 0.06 & 0.99 & 0.04 \\
\hline & 2001-10-07 & 1.02 & 0.03 & 0.93 & 0.03 \\
\hline & 2006-10-04 & 0.93 & 0.13 & 1.03 & 0.03 \\
\hline & 2007-02-01 & 0.97 & 0.13 & 0.90 & 0.01 \\
\hline 50522 & 1998-11-26 & 1.11 & 0.05 & 1.16 & 0.04 \\
\hline 57478 & 1998-11-27 & 1.14 & 0.07 & 1.14 & 0.06 \\
\hline 59148 & $1998-11-28$ & 1.09 & 0.06 & 1.00 & 0.05 \\
\hline \multirow[t]{2}{*}{62345} & $1998-11-30$ & 1.21 & 0.03 & 1.19 & 0.03 \\
\hline & 2006-10-03 & 1.09 & 0.04 & 1.18 & 0.03 \\
\hline 62898 & 1998-11-26 & 0.58 & 0.06 & 0.62 & 0.05 \\
\hline 74874 & $1998-11-28$ & 1.03 & 0.05 & 1.05 & 0.04 \\
\hline \multirow[t]{2}{*}{78235} & $1998-11-28$ & 1.11 & 0.04 & 1.11 & 0.03 \\
\hline & 2007-03-30 & 1.08 & 0.04 & 1.15 & 0.04 \\
\hline \multirow[t]{3}{*}{82635} & 1998-11-30 & 1.05 & 0.02 & 1.08 & 0.02 \\
\hline & 2007-03-12 & 1.07 & 0.07 & 1.02 & 0.07 \\
\hline & 2007-03-30 & 1.03 & 0.03 & 1.04 & 0.03 \\
\hline
\end{tabular}


Table 1-Continued

\begin{tabular}{|c|c|c|c|c|c|}
\hline $\begin{array}{l}\text { Star } \\
(H D)\end{array}$ & Date & $\begin{array}{c}(V / R)_{H} \\
(\mathrm{H})\end{array}$ & $\begin{array}{c}\text { error } \\
\left(\epsilon_{H}\right)\end{array}$ & $\begin{array}{c}(V / R)_{K} \\
(\mathrm{~K})\end{array}$ & $\begin{array}{r}\text { error } \\
\left(\epsilon_{K}\right)\end{array}$ \\
\hline \multirow[t]{2}{*}{85444} & 1998-11-30 & 1.11 & 0.05 & 1.23 & 0.06 \\
\hline & 2007-03-30 & 1.09 & 0.04 & 0.99 & 0.07 \\
\hline \multirow[t]{2}{*}{93813} & 1998-11-30 & 1.16 & 0.06 & 1.17 & 0.05 \\
\hline & 2007-03-11 & 1.08 & 0.06 & 1.17 & 0.14 \\
\hline \multirow[t]{2}{*}{98262} & $2005-06-27$ & 1.05 & 0.06 & 1.01 & 0.06 \\
\hline & $2007-05-27$ & 1.01 & 0.03 & 1.00 & 0.04 \\
\hline \multirow[t]{4}{*}{105707} & 2004-05-28 & 1.14 & 0.04 & 1.01 & 0.04 \\
\hline & 2004-06-29 & 0.99 & 0.06 & 1.14 & 0.11 \\
\hline & 2005-06-26 & 1.15 & 0.05 & 0.93 & 0.04 \\
\hline & 2008-06-22 & 1.11 & 0.02 & 1.04 & 0.02 \\
\hline \multirow[t]{4}{*}{124294} & 2004-05-28 & 1.12 & 0.04 & 0.92 & 0.04 \\
\hline & $2005-06-25$ & 0.98 & 0.05 & 0.93 & 0.06 \\
\hline & 2007-05-28 & 1.08 & 0.04 & 0.96 & 0.08 \\
\hline & 2008-06-22 & 0.98 & 0.03 & 0.90 & 0.04 \\
\hline \multirow[t]{3}{*}{124547} & 2004-05-28 & 1.19 & 0.05 & 0.85 & 0.06 \\
\hline & 2004-06-29 & 1.00 & 0.05 & 1.00 & 0.06 \\
\hline & $2005-06-26$ & 0.98 & 0.05 & 0.90 & 0.06 \\
\hline 131430 & 2004-07-01 & 1.28 & 0.15 & $\ldots$ & $\ldots$ \\
\hline \multirow[t]{3}{*}{139063} & 2004-05-28 & 0.92 & 0.05 & 0.81 & 0.06 \\
\hline & 2004-06-30 & 1.05 & 0.09 & 1.05 & 0.12 \\
\hline & $2004-07-21$ & 1.20 & 0.08 & 1.07 & 0.12 \\
\hline \multirow[t]{2}{*}{140227} & $2004-07-20$ & 1.04 & 0.07 & 0.97 & 0.03 \\
\hline & 2007-05-28 & 1.24 & 0.07 & 1.08 & 0.18 \\
\hline 145849 & 2004-06-29 & 0.63 & 0.05 & $\cdots$ & $\ldots$ \\
\hline 153727 & $2004-07-21$ & 1.11 & 0.18 & $\ldots$ & $\ldots$ \\
\hline 153834 & 2004-07-01 & 1.32 & 0.05 & $\cdots$ & $\cdots$ \\
\hline \multirow[t]{4}{*}{156283} & 2004-09-03 & 1.14 & 0.03 & 1.08 & 0.05 \\
\hline & 2005-06-27 & 1.19 & 0.05 & 0.89 & 0.03 \\
\hline & 2007-03-11 & 0.95 & 0.04 & 1.02 & 0.05 \\
\hline & $2007-05-27$ & 1.11 & 0.04 & 1.00 & 0.07 \\
\hline
\end{tabular}


Table 1-Continued

\begin{tabular}{lccccc}
\hline \hline $\begin{array}{c}\text { Star } \\
(\mathrm{HD})\end{array}$ & Date & $\begin{array}{c}(V / R)_{H} \\
(\mathrm{H})\end{array}$ & $\begin{array}{c}\text { error } \\
\left(\epsilon_{H}\right)\end{array}$ & $\begin{array}{c}(V / R)_{K} \\
(\mathrm{~K})\end{array}$ & $\begin{array}{c}\text { error } \\
\left(\epsilon_{K}\right)\end{array}$ \\
\hline & $2008-06-22$ & 1.07 & 0.01 & 0.99 & 0.02 \\
\hline 161832 & $2004-06-30$ & 1.12 & 0.04 & $\cdots$ & $\cdots$ \\
\hline 163770 & $2004-05-27$ & 0.97 & 0.03 & 0.82 & 0.04 \\
& $2005-06-26$ & 0.93 & 0.03 & 0.72 & 0.05 \\
\hline 168454 & $2004-05-27$ & 1.18 & 0.11 & 1.25 & 0.12 \\
& $2004-07-20$ & 1.16 & 0.06 & 1.13 & 0.08 \\
& $2004-09-03$ & 1.32 & 0.08 & 1.11 & 0.08 \\
& $2005-06-25$ & 1.15 & 0.07 & 1.08 & 0.08 \\
& $2007-05-27$ & 1.12 & 0.05 & 0.94 & 0.06 \\
& $2008-06-22$ & 1.17 & 0.06 & 1.10 & 0.08 \\
\hline 171443 & $2004-05-28$ & 0.99 & 0.05 & 0.94 & 0.04 \\
& $2004-06-29$ & 1.01 & 0.06 & 0.94 & 0.05 \\
& $2005-06-25$ & 1.12 & 0.04 & 1.00 & 0.05 \\
& $2006-08-31$ & 1.01 & 0.05 & 1.01 & 0.04 \\
\hline 175443 & $2004-07-20$ & 1.12 & 0.06 & 1.37 & 0.08 \\
& $2005-06-26$ & 1.10 & 0.18 & 0.97 & 0.07 \\
\hline 177199 & $2004-07-21$ & 0.82 & 0.16 & $\cdots$ & $\cdots$ \\
\hline 183387 & $2004-07-01$ & 1.30 & 0.08 & $\cdots$ & $\cdots$ \\
\hline 183439 & $2001-10-07$ & 0.67 & 0.07 & 0.65 & 0.06 \\
\hline 184835 & $2001-10-03$ & 1.23 & 0.07 & 1.14 & 0.06 \\
\hline 187372 & $1998-11-29$ & 0.68 & 0.10 & 0.56 & 0.08 \\
\hline 190327 & $1998-11-28$ & 1.10 & 0.06 & 1.11 & 0.05 \\
\hline 190940 & $2001-10-08$ & 1.02 & 0.04 & 1.04 & 0.04 \\
& $2004-05-28$ & 1.25 & 0.05 & 1.04 & 0.04 \\
& $2004-06-29$ & 1.16 & 0.04 & 0.97 & 0.03 \\
& $2005-06-25$ & 1.34 & 0.07 & 1.15 & 0.07 \\
& $2006-08-31$ & 1.02 & 0.03 & 1.03 & 0.04 \\
& $2008-06-22$ & 1.09 & 0.03 & 1.04 & 0.04 \\
\hline 194317 & $2005-06-27$ & 1.26 & 0.06 & $\cdots$ & $\cdots$ \\
\hline 196574 & $1998-11-26$ & 1.17 & 0.06 & 1.17 & 0.05 \\
& & & & & \\
\hline 10
\end{tabular}


Table 1-Continued

\begin{tabular}{|c|c|c|c|c|c|}
\hline $\begin{array}{l}\text { Star } \\
(\mathrm{HD})\end{array}$ & Date & $\begin{array}{c}(V / R)_{H} \\
(\mathrm{H})\end{array}$ & $\begin{array}{c}\text { error } \\
\left(\epsilon_{H}\right)\end{array}$ & $\begin{array}{c}(V / R)_{K} \\
(\mathrm{~K})\end{array}$ & $\begin{array}{r}\text { error } \\
\left(\epsilon_{K}\right)\end{array}$ \\
\hline 198026 & $2001-10-04$ & 0.91 & 0.04 & 0.76 & 0.04 \\
\hline \multirow[t]{3}{*}{198134} & 2001-10-08 & 1.21 & 0.05 & 1.13 & 0.04 \\
\hline & 2004-06-30 & 1.22 & 0.06 & 1.05 & 0.06 \\
\hline & 2006-08-31 & 1.10 & 0.05 & 1.06 & 0.05 \\
\hline 199169 & 2001-10-03 & 1.06 & 0.03 & 0.85 & 0.03 \\
\hline 199253 & $1998-11-27$ & 1.06 & 0.04 & 1.05 & 0.04 \\
\hline 200644 & $2001-10-04$ & 0.75 & 0.05 & 0.63 & 0.05 \\
\hline 202951 & $1998-10-26$ & 0.90 & 0.06 & 0.95 & 0.05 \\
\hline \multirow[t]{2}{*}{203387} & 2006-10-05 & 0.92 & 0.03 & 1.01 & 0.04 \\
\hline & 2008-06-22 & 1.06 & 0.02 & 1.06 & 0.02 \\
\hline 204724 & 2001-10-05 & 0.70 & 0.02 & 0.58 & 0.02 \\
\hline \multirow[t]{2}{*}{205435} & $1998-10-27$ & 1.17 & 0.02 & 1.12 & 0.02 \\
\hline & 2006-10-05 & 1.05 & 0.03 & 1.07 & 0.03 \\
\hline 209747 & 2001-10-07 & 0.97 & 0.06 & 0.76 & 0.05 \\
\hline 210434 & 1998-10-25 & 1.15 & 0.05 & 1.13 & 0.04 \\
\hline \multirow[t]{6}{*}{211073} & 2001-10-03 & 1.06 & 0.02 & 0.98 & 0.02 \\
\hline & 2004-06-29 & 1.04 & 0.03 & 0.91 & 0.03 \\
\hline & 2004-07-20 & 1.04 & 0.07 & 1.02 & 0.05 \\
\hline & $2005-06-26$ & 1.20 & 0.04 & 0.99 & 0.03 \\
\hline & 2006-08-31 & 1.06 & 0.03 & 0.97 & 0.04 \\
\hline & 2008-06-23 & 1.06 & 0.03 & 0.97 & 0.04 \\
\hline \multirow[t]{2}{*}{211391} & $2006-10-03$ & 1.16 & 0.06 & 1.18 & 0.03 \\
\hline & $2008-06-23$ & 1.16 & 0.02 & 1.15 & 0.03 \\
\hline \multirow[t]{3}{*}{214868} & $2004-06-30$ & 1.22 & 0.05 & 1.02 & 0.05 \\
\hline & $2005-06-26$ & 1.31 & 0.06 & 1.10 & 0.04 \\
\hline & 2006-09-01 & 1.12 & 0.04 & 1.03 & 0.05 \\
\hline \multirow[t]{4}{*}{215167} & $2001-10-07$ & 1.00 & 0.04 & 0.82 & 0.03 \\
\hline & 2004-07-01 & 1.10 & 0.05 & 0.92 & 0.05 \\
\hline & $2005-06-27$ & 0.88 & 0.16 & 0.72 & 0.08 \\
\hline & 2006-09-01 & 0.97 & 0.05 & 0.89 & 0.06 \\
\hline
\end{tabular}


Table 1-Continued

\begin{tabular}{lccccc}
\hline \hline $\begin{array}{l}\text { Star } \\
\text { (HD) }\end{array}$ & Date & $\begin{array}{c}(V / R)_{H} \\
(\mathrm{H})\end{array}$ & $\begin{array}{c}\text { error } \\
\left(\epsilon_{H}\right)\end{array}$ & $\begin{array}{c}(V / R)_{K} \\
(\mathrm{~K})\end{array}$ & $\begin{array}{c}\text { error } \\
\left(\epsilon_{K}\right)\end{array}$ \\
\hline 216446 & $2001-10-08$ & 1.14 & 0.04 & 1.14 & 0.04 \\
\hline 216718 & $1998-11-26$ & 1.08 & 0.08 & 1.13 & 0.07 \\
\hline 217382 & $2001-10-08$ & 1.06 & 0.04 & 1.04 & 0.04 \\
\hline 217906 & $2001-10-03$ & 0.81 & 0.01 & 0.73 & 0.01 \\
\hline 218452 & $2001-10-07$ & 1.10 & 0.03 & 1.10 & 0.03 \\
\hline 218527 & $1998-11-26$ & 1.24 & 0.06 & 1.30 & 0.06 \\
\hline 219916 & $1998-10-26$ & 1.05 & 0.06 & 1.19 & 0.05 \\
\hline 220363 & $2001-10-07$ & 1.10 & 0.04 & 1.10 & 0.04 \\
\hline 221673 & $2004-07-20$ & 1.09 & 0.11 & 0.91 & 0.04 \\
\hline 222404 & $1998-10-26$ & 1.10 & 0.04 & 1.08 & 0.03 \\
& $2006-09-01$ & 1.10 & 0.04 & 1.09 & 0.04 \\
& $2006-10-04$ & 1.28 & 0.05 & 1.25 & 0.04 \\
\hline 222643 & $2001-10-03$ & 1.16 & 0.09 & $\ldots$ & $\ldots$ \\
\hline 222764 & $2001-10-04$ & 0.82 & 0.04 & 0.71 & 0.04 \\
\hline 223460 & $1998-11-29$ & 1.07 & 0.03 & 1.07 & 0.03 \\
\hline 224533 & $1998-10-26$ & 1.07 & 0.03 & 1.13 & 0.03 \\
\hline
\end{tabular}


Table 2. Summary of Sample

\begin{tabular}{|c|c|c|c|c|c|c|c|}
\hline $\begin{array}{l}\text { Star } \\
\text { (HD) }\end{array}$ & $B-V$ & $\begin{array}{c}M_{V} \\
(\mathrm{mag})\end{array}$ & $\begin{array}{c}\langle V / R\rangle(\sigma) \\
(\mathrm{H})\end{array}$ & $\begin{array}{c}\langle V / R\rangle(\sigma) \\
\quad(\mathrm{K})\end{array}$ & {$[\mathrm{Fe} / \mathrm{H}]^{\mathrm{a}}$} & $\begin{array}{c}v_{r} \\
\mathrm{~km} \mathrm{~s}^{-1}\end{array}$ & $\begin{array}{c}A_{V} \\
\text { mag }\end{array}$ \\
\hline 1013 & 1.573 & -0.2 & $0.79(0.03)$ & $0.70(0.03)$ & $\ldots$ & -46.6 & 0.029 \\
\hline 1227 & 0.917 & 0.45 & $1.15(0.07)$ & $1.13(0.06)$ & $\cdots$ & 1.4 & 0.052 \\
\hline 1522 & 1.213 & -1.2 & $1.14(0.05)$ & $1.03(0.03)$ & -0.09 & 18.6 & 0.020 \\
\hline 3627 & 1.278 & 0.8 & $1.096(0.018)$ & $1.094(0.024)$ & 0.04 & -7.3 & 0.000 \\
\hline 4128 & 1.023 & -0.3 & $1.049(0.017)$ & $1.095(0.021)$ & -0.09 & 13.0 & 0.000 \\
\hline 4482 & 0.973 & 0.98 & $1.11(0.03)$ & $1.10(0.03)$ & & -0.8 & 0.004 \\
\hline 4730 & 1.312 & 0.59 & $1.06(0.06)$ & $1.06(0.05)$ & -0.03 & 3.5 & 0.034 \\
\hline 5516 & 0.940 & 0.06 & $1.10(0.04)$ & $1.22(0.03)$ & -0.54 & -10.3 & 0.000 \\
\hline 6953 & 1.466 & 0.59 & $1.14(0.05)$ & $1.08(0.04)$ & $\ldots$ & 3.2 & 0.017 \\
\hline 7318 & 1.035 & -0.67 & $1.16(0.06)$ & $1.14(0.05)$ & -0.08 & 5.9 & 0.020 \\
\hline 9138 & 1.372 & -0.37 & $0.84(0.041)$ & $0.928(0.037)$ & -0.39 & 34.2 & 0.033 \\
\hline 9774 & 0.960 & -0.4 & $1.16(0.06)$ & $1.18(0.06)$ & -0.1 & -4.0 & 0.062 \\
\hline 11559 & 0.941 & 0.79 & $1.13(0.05)$ & $1.08(0.04)$ & -0.11 & 30.3 & 0.000 \\
\hline 11930 & 1.430 & -0.55 & $1.11(0.05)$ & $1.00(0.04)$ & -0.18 & 26.7 & 0.031 \\
\hline 14652 & 1.652 & -2.73 & $0.62(0.05)$ & $0.61(0.05)$ & $\ldots$ & 22.8 & 0.094 \\
\hline 15656 & 1.470 & -0.24 & $0.77(0.04)$ & $0.62(0.04)$ & -0.16 & -35.9 & 0.057 \\
\hline 15694 & 1.255 & -0.56 & $1.06(0.05)$ & $1.01(0.04)$ & -0.17 & 25.6 & 0.021 \\
\hline 16058 & 1.652 & $\cdots$ & $0.55(0.03)$ & $0.42(0.02)$ & $\cdots$ & -7.1 & $\ldots$ \\
\hline 17506 & 1.690 & -4.29 & $0.64(0.06)$ & $0.61(0.05)$ & $\ldots$ & -1.1 & 0.573 \\
\hline 19349 & 1.595 & -0.81 & $1.04(0.04)$ & $0.98(0.03)$ & $\ldots$ & 16.7 & 0.041 \\
\hline 19476 & 0.980 & 1.12 & $1.16(0.04)$ & $1.22(0.04)$ & 0.04 & 29.2 & 0.000 \\
\hline 20610 & 0.904 & 0.41 & $1.19(0.08)$ & $1.15(0.07)$ & -0.07 & 23.9 & 0.003 \\
\hline 20893 & 1.229 & 0.14 & $0.91(0.04)$ & $1.11(0.04)$ & 0.02 & 3.0 & 0.020 \\
\hline 21051 & 1.230 & 0.28 & $1.13(0.07)$ & $1.18(0.06)$ & $\cdots$ & 18.6 & 0.004 \\
\hline 21552 & 1.367 & -0.81 & $1.01(0.03)$ & $0.94(0.03)$ & -0.24 & 14.4 & 0.037 \\
\hline 26076 & 1.012 & 0.78 & $1.30(0.10)$ & $1.28(0.09)$ & $\ldots$ & -5.5 & 0.023 \\
\hline 26846 & 1.162 & 0.86 & $1.11(0.04)$ & $1.10(0.03)$ & 0.09 & 6.8 & 0.000 \\
\hline 27278 & 0.955 & 0.79 & $1.03(0.05)$ & $1.08(0.05)$ & $\cdots$ & 24.1 & 0.049 \\
\hline 27371 & 0.987 & 0.28 & $1.09(0.03)$ & $1.10(0.03)$ & -0.02 & 38.7 & 0.000 \\
\hline 27697 & 0.983 & 0.4 & $1.132(0.036)$ & $1.202(0.035)$ & 0 & 38.8 & 0.000 \\
\hline
\end{tabular}


Table 2-Continued

\begin{tabular}{ccrrrrrr}
\hline \hline $\begin{array}{c}\text { Star } \\
(\mathrm{HD})\end{array}$ & $B-V$ & $\begin{array}{c}M_{V} \\
(\mathrm{mag})\end{array}$ & \multicolumn{1}{c}{$\langle\mathrm{V} / R\rangle(\sigma)$} & $\begin{array}{c}\langle V / R\rangle(\sigma) \\
(\mathrm{H})\end{array}$ & $\begin{array}{r}\mathrm{Fe} / \mathrm{H}]^{\mathrm{a}} \\
\mathrm{km} \mathrm{s}\end{array}$ & $\begin{array}{c}v_{r} \\
\mathrm{kmag}\end{array}$ \\
\hline 28305 & 1.014 & 0.15 & $1.173(0.023)$ & $1.070(0.021)$ & 0.04 & 38.2 & 0.000 \\
28307 & 0.951 & 0.42 & $1.07(0.03)$ & $1.13(0.02)$ & 0.04 & 39.8 & 0.000 \\
32357 & 1.132 & -0.33 & $1.05(0.03)$ & $1.12(0.02)$ & $\ldots$ & -8.0 & 0.210 \\
32887 & 1.472 & -1.04 & $0.94(0.03)$ & $0.96(0.03)$ & -0.17 & 1.0 & 0.000 \\
33856 & 1.185 & -0.65 & $1.07(0.06)$ & $1.17(0.05)$ & 0.06 & 41.0 & 0.025 \\
35186 & 1.416 & -0.99 & $1.22(0.04)$ & $1.23(0.04)$ & -0.03 & -19.7 & 0.095 \\
42633 & 1.340 & -0.57 & $1.04(0.05)$ & $1.07(0.05)$ & 0 & 8.6 & 0.089 \\
42995 & 1.586 & -1.87 & $0.38(0.05)$ & $0.39(0.04)$ & $\ldots$ & 19.0 & 0.032 \\
49161 & 1.393 & -1.1 & $1.026(0.026)$ & $0.918(0.009)$ & -0.03 & 46.2 & 0.049 \\
50522 & 0.849 & 0.76 & $1.11(0.05)$ & $1.16(0.04)$ & 0.05 & 8.9 & 0.000 \\
57478 & 0.974 & -0.71 & $1.14(0.07)$ & $1.14(0.06)$ & $\ldots$ & 13.2 & 0.039 \\
59148 & 1.117 & -0.34 & $1.09(0.06)$ & $1.00(0.05)$ & $\ldots$ & 36.0 & 0.000 \\
62345 & 0.932 & 0.35 & $1.167(0.024)$ & $1.185(0.021)$ & -0.16 & 20.6 & 0.000 \\
62898 & 1.594 & -1.04 & $0.58(0.06)$ & $0.62(0.05)$ & $\ldots$ & -13.4 & 0.033 \\
74874 & 0.684 & 0.29 & $1.03(0.05)$ & $1.05(0.04)$ & -0.14 & 36.4 & 0.000 \\
78235 & 0.888 & 0.95 & $1.095(0.028)$ & $1.124(0.024)$ & $\ldots$ & -13.1 & 0.000 \\
82635 & 0.916 & 0.89 & $1.045(0.016)$ & $1.065(0.016)$ & -0.15 & -11.7 & 0.000 \\
85444 & 0.928 & -0.51 & $1.098(0.031)$ & $1.128(0.046)$ & -0.14 & -14.5 & 0.006 \\
93813 & 1.245 & -0.14 & $1.120(0.042)$ & $1.170(0.074)$ & -0.3 & -1.2 & 0.000 \\
98262 & 1.398 & -2.08 & $1.018(0.027)$ & $1.003(0.033)$ & -0.2 & -9.6 & 0.015 \\
105707 & 1.331 & -1.84 & $1.110(0.016)$ & $1.019(0.016)$ & -0.13 & 4.9 & 0.016 \\
124294 & 1.336 & 0 & $1.037(0.019)$ & $0.918(0.024)$ & -0.39 & -4.0 & 0.000 \\
124547 & 1.368 & -1.06 & $1.057(0.029)$ & $0.917(0.035)$ & 0.17 & 10.5 & 0.048 \\
131430 & 1.331 & $\ldots$ & $1.28(0.15)$ & $\ldots$ & 0.04 & 8.8 & $\ldots$ \\
139063 & 1.380 & -0.28 & $1.008(0.038)$ & $0.893(0.049)$ & -0.18 & -24.9 & 0.000 \\
140227 & 1.362 & -1.55 & $1.140(0.049)$ & $0.973(0.030)$ & $\ldots$ & -30.4 & 0.060 \\
145849 & 1.340 & $\ldots$ & $0.63(0.05)$ & $\ldots$ & $\ldots$ & -30.6 & $\ldots$ \\
153727 & 1.355 & $\ldots$ & $1.11(0.18)$ & $\ldots$ & $\ldots$ & 44.2 & $\ldots$ \\
153834 & $\ldots$ & $\ldots$ & $1.32(0.05)$ & $\ldots$ & $\ldots$ & 11.3 & $\ldots$ \\
156283 & 1.437 & -2.09 & $1.076(0.009)$ & $0.977(0.015)$ & -0.18 & -25.6 & 0.041 \\
& & & & & & &
\end{tabular}


Table 2-Continued

\begin{tabular}{|c|c|c|c|c|c|c|c|}
\hline $\begin{array}{l}\text { Star } \\
\text { (HD) }\end{array}$ & $B-V$ & $\begin{array}{c}M_{V} \\
(\mathrm{mag})\end{array}$ & $\begin{array}{c}\langle V / R\rangle(\sigma) \\
\quad(\mathrm{H})\end{array}$ & $\begin{array}{c}\langle V / R\rangle(\sigma) \\
\quad(\mathrm{K})\end{array}$ & {$[\mathrm{Fe} / \mathrm{H}]^{\mathrm{a}}$} & $\begin{array}{c}v_{r} \\
\mathrm{~km} \mathrm{~s}^{-1}\end{array}$ & $\begin{array}{c}A_{V} \\
\text { mag }\end{array}$ \\
\hline 161832 & 1.390 & . & $1.12(0.04)$ & & .. & -32 & \\
\hline 163770 & 1.351 & -2.7 & $0.950(0.021)$ & $0.781(0.031)$ & -0.24 & -28.3 & 0.080 \\
\hline 168454 & 1.376 & -2.16 & $1.168(0.027)$ & $1.068(0.032)$ & -0.01 & -19.9 & 0.024 \\
\hline 171443 & 1.333 & 0.21 & $1.046(0.024)$ & $0.973(0.022)$ & -0.18 & 35.8 & 0.000 \\
\hline 175443 & 1.349 & 0.15 & $1.118(0.057)$ & $1.143(0.053)$ & $\ldots$ & 13.3 & 0.036 \\
\hline 177199 & 1.339 & $\cdots$ & $0.82(0.16)$ & $\ldots$ & .. & -7.0 & $\ldots$ \\
\hline 183387 & 1.318 & $\cdots$ & $1.30(0.08)$ & $\ldots$ & $\cdots$ & $\ldots$ & $\ldots$ \\
\hline 183439 & 1.502 & -0.35 & $0.67(0.07)$ & $0.65(0.06)$ & $\ldots$ & -85.5 & 0.014 \\
\hline 184835 & 1.242 & 0 & $1.23(0.07)$ & $1.14(0.06)$ & $\cdots$ & -7.3 & 0.212 \\
\hline 187372 & 1.640 & -1.76 & $0.68(0.10)$ & $0.56(0.08)$ & .. & 3 & 0.370 \\
\hline 190327 & 1.062 & -0.52 & $1.10(0.06)$ & $1.11(0.05)$ & -0.15 & -29.5 & 0.177 \\
\hline 190940 & 1.313 & -0.97 & $1.099(0.016)$ & $1.024(0.016)$ & 0.03 & -9.8 & 0.027 \\
\hline 194317 & 1.330 & $\ldots$ & $1.26(0.06)$ & & -0.17 & -14.6 & $\ldots$ \\
\hline 196574 & 0.949 & -1.03 & $1.17(0.06)$ & $1.17(0.05)$ & -0.13 & -5.6 & 0.065 \\
\hline 198026 & 1.645 & -1.24 & $0.91(0.04)$ & $0.76(0.04)$ & $\ldots$ & -22.0 & 0.090 \\
\hline 198134 & 1.299 & -0.5 & $1.172(0.030)$ & $1.091(0.028)$ & -0.12 & -24.1 & 0.038 \\
\hline 199169 & 1.480 & -1.76 & $1.06(0.03)$ & $0.85(0.03)$ & -0.16 & 8.1 & 0.115 \\
\hline 199253 & 1.119 & -0.64 & $1.06(0.04)$ & $1.05(0.04)$ & -0.18 & -11.2 & 0.049 \\
\hline 200644 & 1.650 & -1.02 & $0.75(0.05)$ & $0.63(0.05)$ & ... & -15.3 & 0.043 \\
\hline 202951 & 1.648 & -1.97 & $0.90(0.06)$ & $0.95(0.05)$ & $\cdots$ & -37.0 & 0.099 \\
\hline 203387 & 0.899 & 0.18 & $1.017(0.017)$ & $1.050(0.018)$ & -0.23 & 11.5 & 0.000 \\
\hline 204724 & 1.618 & -1.11 & $0.70(0.02)$ & $0.58(0.02)$ & ... & -18.9 & 0.050 \\
\hline 205435 & 0.887 & 1.1 & $1.13(0.017)$ & $1.105(0.017)$ & -0.31 & 6.9 & 0.000 \\
\hline 209747 & 1.452 & 0.3 & $0.97(0.06)$ & $0.76(0.05)$ & 0.02 & -18.9 & 0.005 \\
\hline 210434 & 0.983 & 1.33 & $1.15(0.05)$ & $1.13(0.04)$ & $\ldots$ & -18.1 & 0.006 \\
\hline 211073 & 1.385 & -1.69 & $1.069(0.012)$ & $0.970(0.013)$ & 0.02 & -10.6 & 0.094 \\
\hline 211391 & 0.983 & 0.32 & $1.160(0.030)$ & $1.165(0.021)$ & 0.01 & -14.7 & 0.000 \\
\hline 214868 & 1.320 & -0.35 & $1.191(0.028)$ & $1.058(0.026)$ & -0.25 & -11.0 & 0.019 \\
\hline 215167 & 1.371 & -1.18 & $1.016(0.026)$ & $0.843(0.023)$ & -0.23 & 21.6 & 0.078 \\
\hline 216446 & 1.257 & -0.23 & $1.14(0.04)$ & $1.14(0.04)$ & -0.19 & -31.9 & 0.030 \\
\hline
\end{tabular}


Table 2-Continued

\begin{tabular}{ccccccrc}
\hline \hline $\begin{array}{c}\text { Star } \\
(\mathrm{HD})\end{array}$ & $B-V$ & $\begin{array}{c}M_{V} \\
(\mathrm{mag})\end{array}$ & $\begin{array}{c}\langle V / R\rangle(\sigma) \\
(\mathrm{H})\end{array}$ & $\begin{array}{c}\langle V / R\rangle(\sigma) \\
(\mathrm{K})\end{array}$ & {$[\mathrm{Fe} / \mathrm{H}]^{\mathrm{a}}$} & $\begin{array}{c}v_{r} \\
\mathrm{~km} \mathrm{~s}{ }^{-1}\end{array}$ & $\begin{array}{c}A_{V} \\
\mathrm{mag}\end{array}$ \\
\hline 216718 & 0.880 & 0.95 & $1.08(0.08)$ & $1.13(0.07)$ & 0.04 & -8.8 & 0.015 \\
217382 & 1.417 & -0.67 & $1.06(0.04)$ & $1.04(0.04)$ & -0.11 & 2.9 & 0.049 \\
217906 & 1.668 & -1.47 & $0.81(0.01)$ & $0.73(0.01)$ & $\ldots$ & 8.0 & 0.000 \\
218452 & 1.409 & 0.22 & $1.10(0.03)$ & $1.10(0.03)$ & -0.02 & -11.6 & 0.032 \\
218527 & 0.906 & 0.73 & $1.24(0.06)$ & $1.30(0.06)$ & -0.31 & -17.8 & 0.009 \\
219916 & 0.837 & 0.7 & $1.05(0.06)$ & $1.19(0.05)$ & -0.07 & -18.4 & 0.000 \\
220363 & 1.316 & 0.07 & $1.10(0.04)$ & $1.10(0.04)$ & -0.01 & -3.9 & 0.018 \\
221673 & 1.385 & -1.16 & $1.09(0.11)$ & $0.91(0.04)$ & -0.03 & -24.7 & 0.090 \\
222404 & 1.030 & 2.51 & $1.144(0.025)$ & $1.128(0.021)$ & 0 & -42.4 & 0.000 \\
222643 & 1.362 & $\ldots$ & $1.16(0.09)$ & $\ldots$ & 0.02 & 6.8 & $\ldots$ \\
222764 & 1.686 & -1.95 & $0.82(0.04)$ & $0.71(0.04)$ & $\ldots$ & -32.7 & 0.060 \\
223460 & 0.793 & 0.25 & $1.07(0.03)$ & $1.07(0.03)$ & $\ldots$ & 0.7 & 0.086 \\
224533 & 0.930 & 0.68 & $1.07(0.03)$ & $1.13(0.03)$ & -0.13 & -0.2 & 0.000 \\
\hline
\end{tabular}

${ }^{a}$ Metallicities taken from McWilliam (1990). 


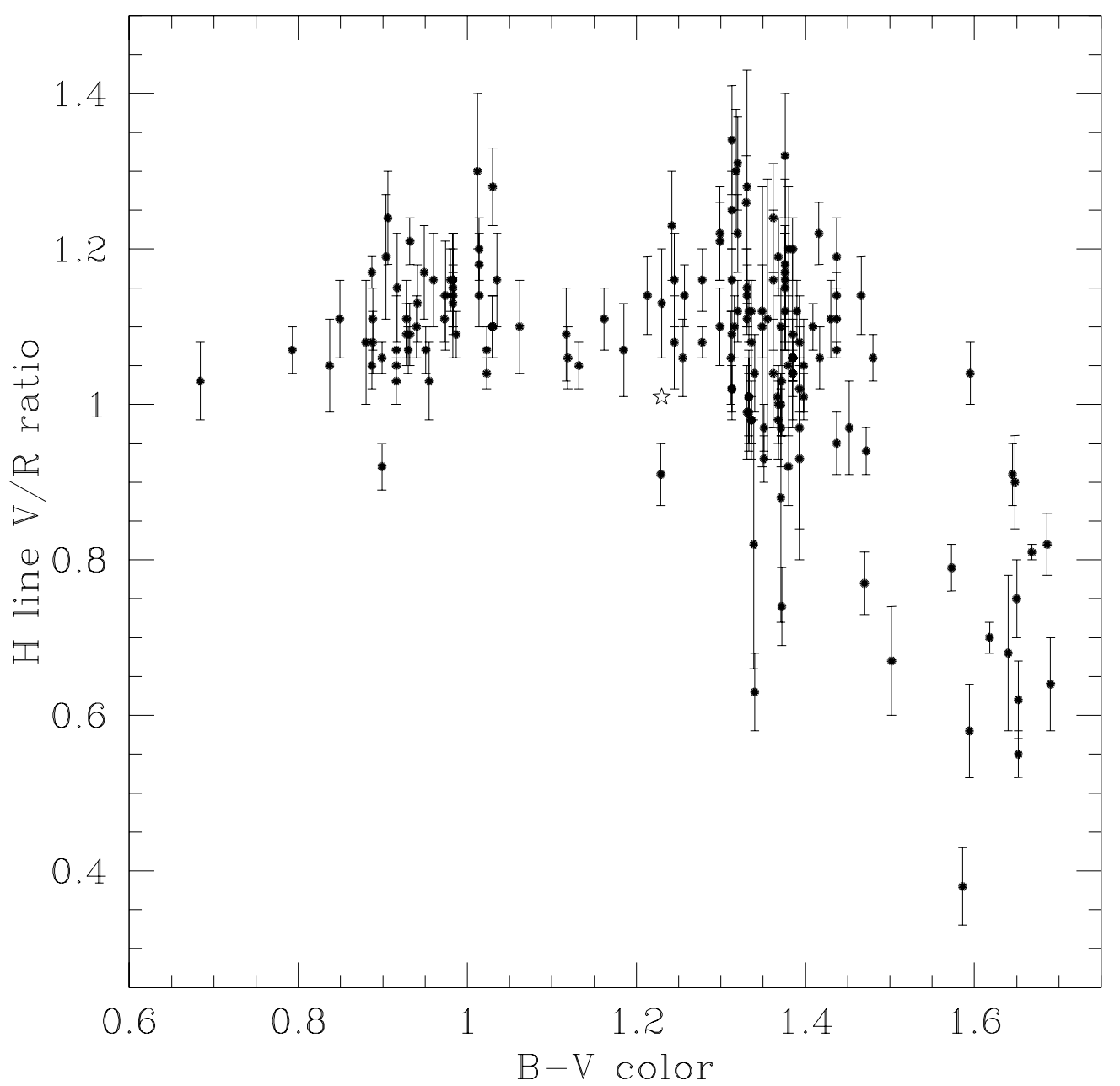

Fig. 1.- The $V / R$ value of the Ca II $\mathrm{H}$ line from Table 1 is plotted versus the $B-V$ color (Table 2). The single star symbol represents Arcturus. Where multiple observations are available for a star, the result for each individual spectrum is shown as a separate data point. Thus a number of stars are represented by multiple points. 


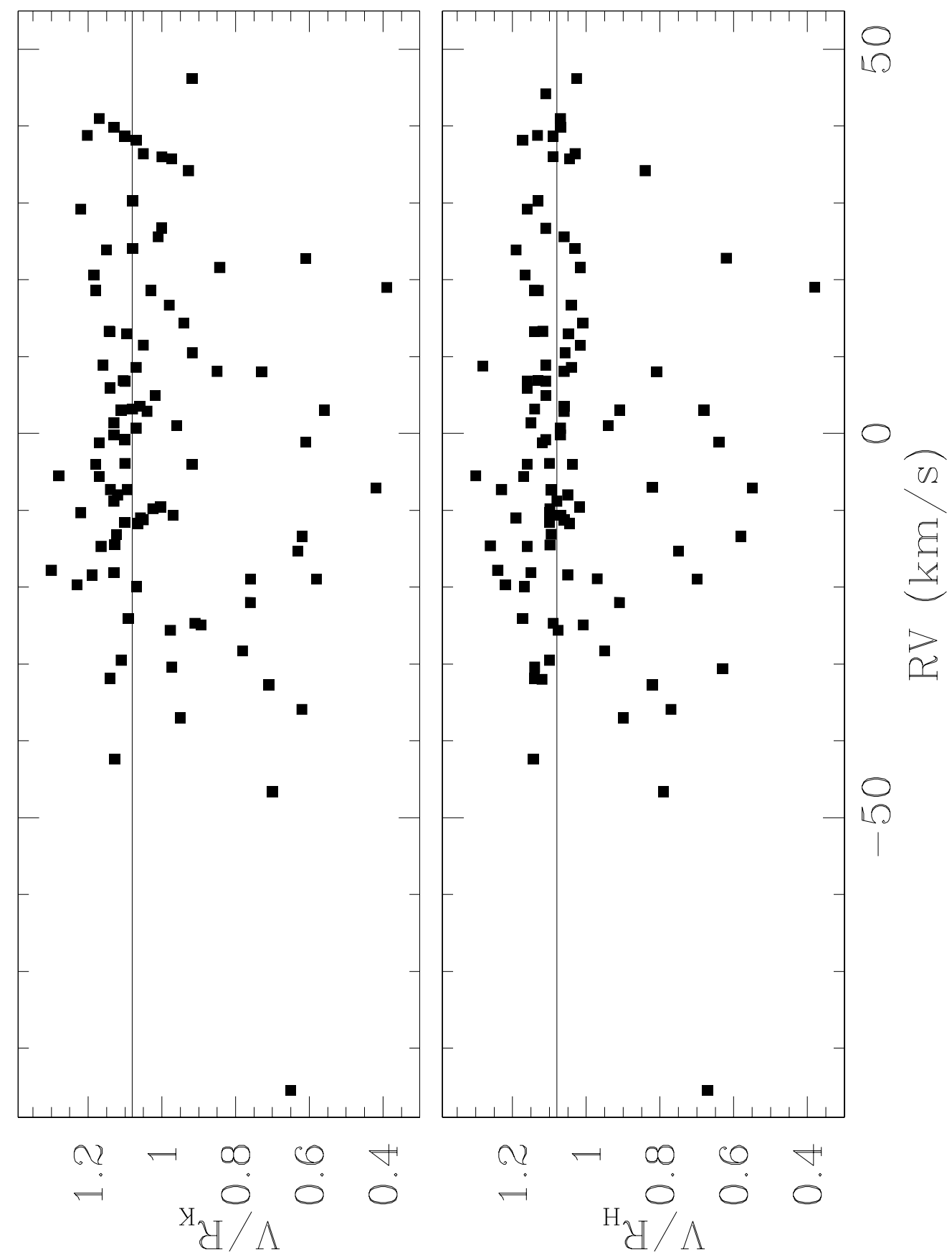

Fig. 2. - The $\mathrm{H}$ and $\mathrm{K}$ line asymmetry parameters $(V / R)_{K}$ and $(V / R)_{H}$ versus heliocentric radial velocity. The lack of correlations in this figure is presented as evidence that the asymmetry trends seen in this, and previous papers, are not dominated by the effects of interstellar absorption. 


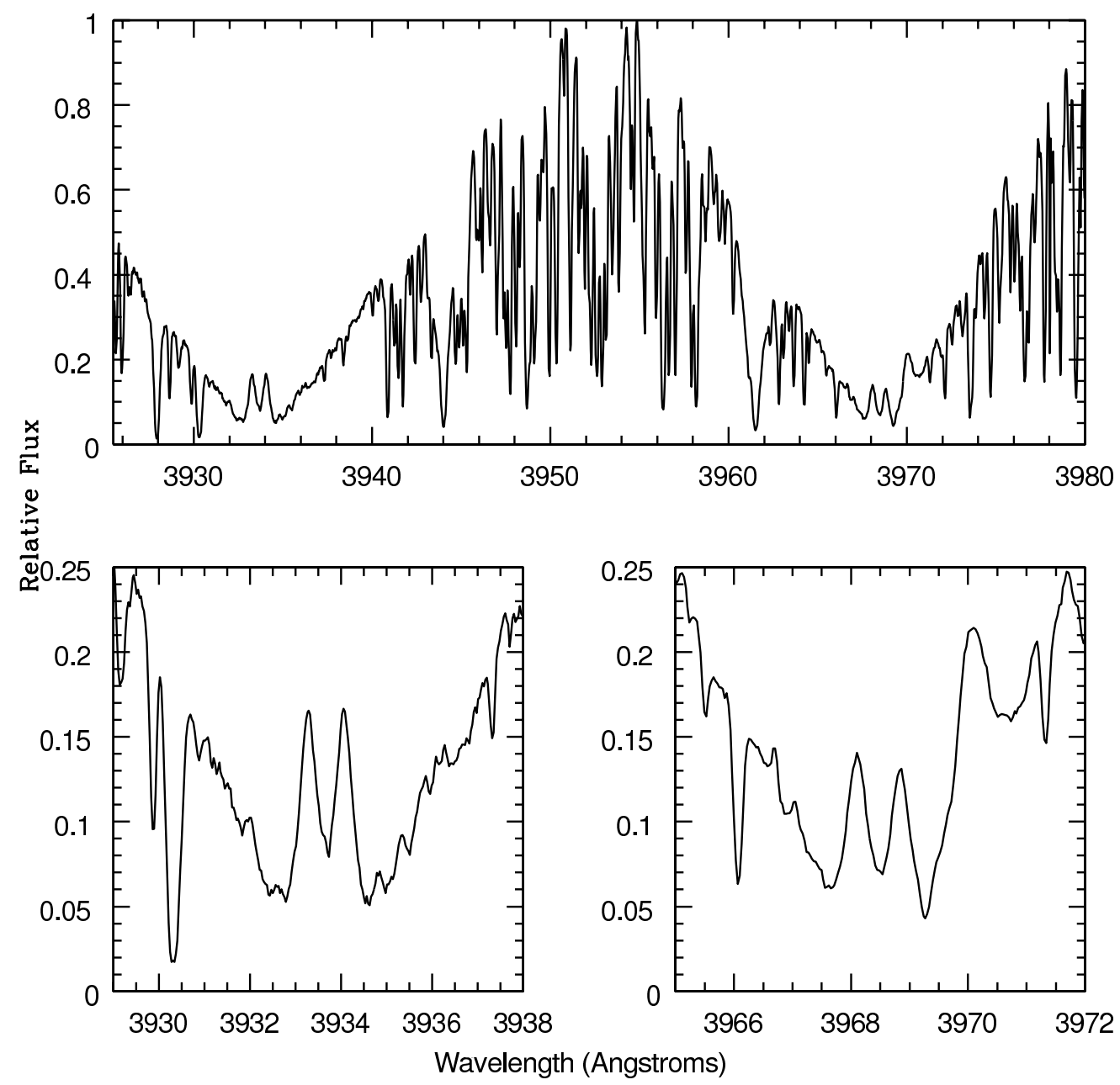

Fig. 3.- The order of the normalized spectrum of HD 156283 that contains the Ca II H and $\mathrm{K}$ lines is plotted in the upper panel. This spectrum was observed on 22 June 2008 using the $2.7 \mathrm{~m}$ telescope at McDonald Observatory. In the bottom left window, an expanded version of the core of the $\mathrm{K}$ line is plotted, to clearly show the near symmetry of the emission peaks. The core of the $\mathrm{H}$ line is plotted in the bottom right window, and reveals the blueward emission peak to be stronger than the redward peak. 


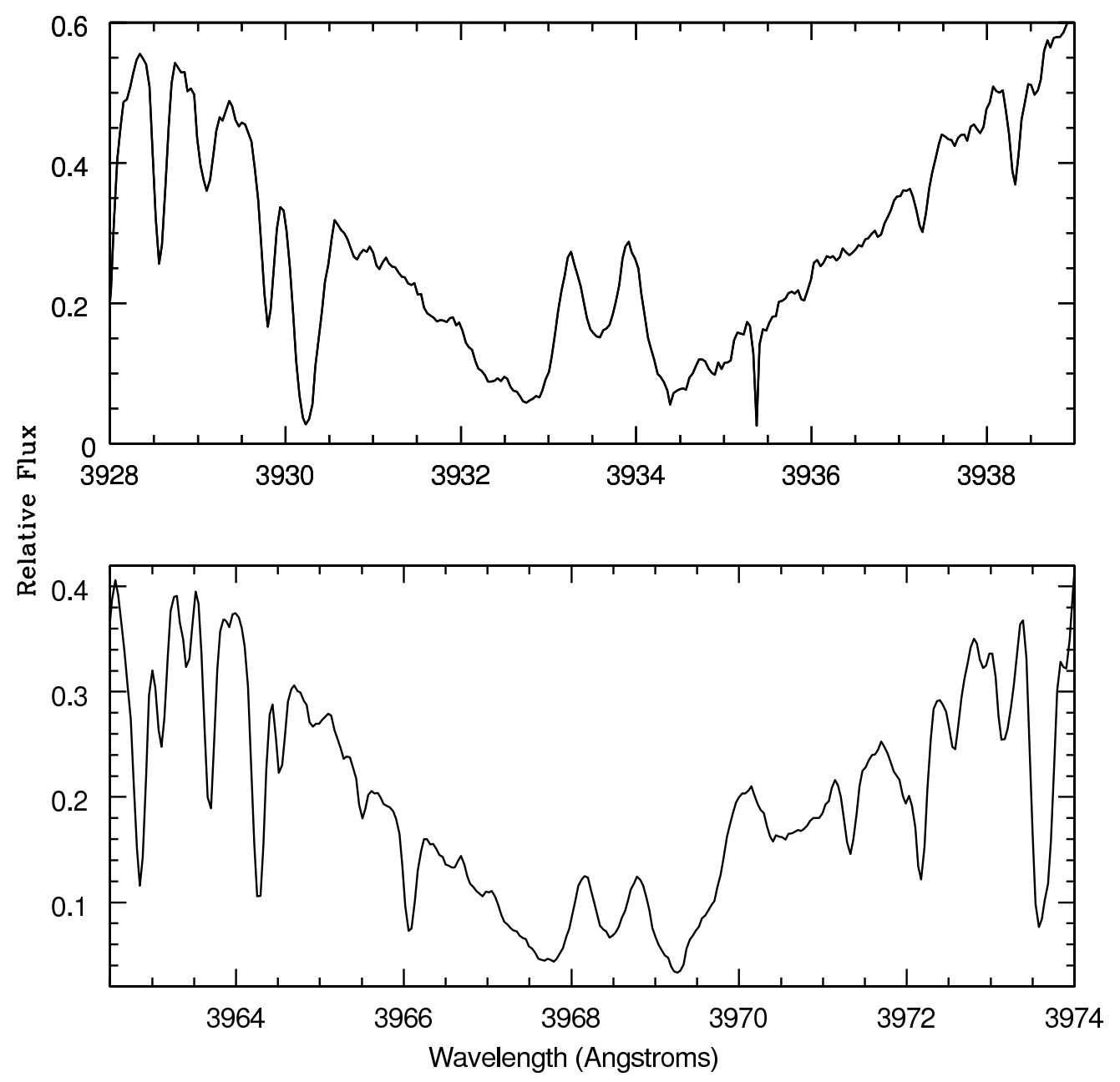

Fig. 4.- The echelle order of the normalized spectrum of HD 21552 containing the Ca II $\mathrm{K}$ line is plotted in the top panel, with the H-line order shown in the bottom window. This spectrum was observed on 3 October 2001 using the $2.1 \mathrm{~m}$ telescope at McDonald Observatory. Whereas the $\mathrm{H}$ emission line is nearly symmetric, the $\mathrm{K}$ line shows a slightly red-enhanced emission profile. 


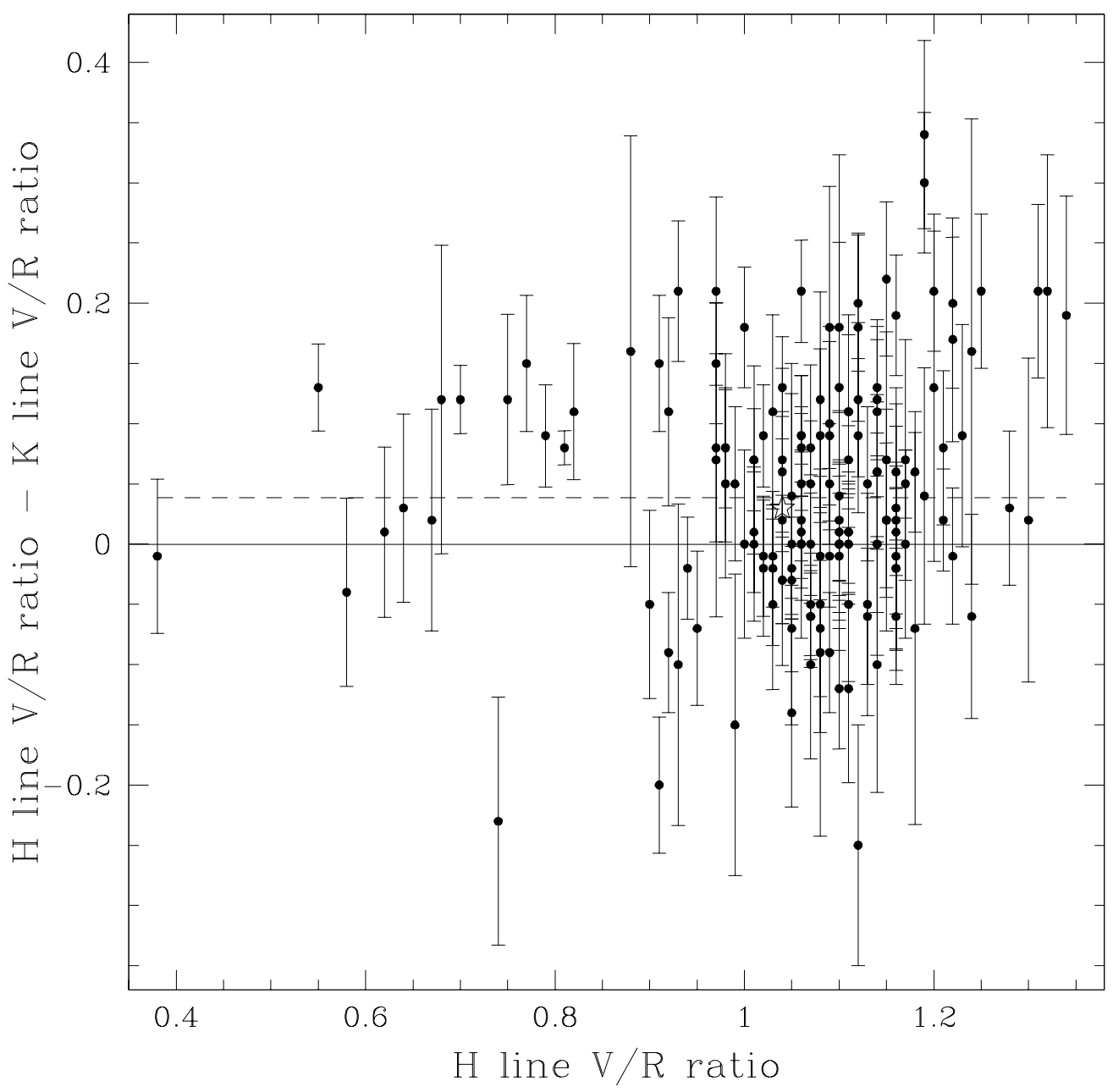

Fig. 5.- The difference between the Ca II $\mathrm{H}$ line and $\mathrm{K}$ line $V / R$ values plotted versus the measured $\mathrm{H}$ line $V / R$. The data are from Table 1 , so certain stars may be represented by multiple points in this diagram. The single star symbol represents Arcturus. The dashed line shows the mean value of the $V / R$ parameter difference. 


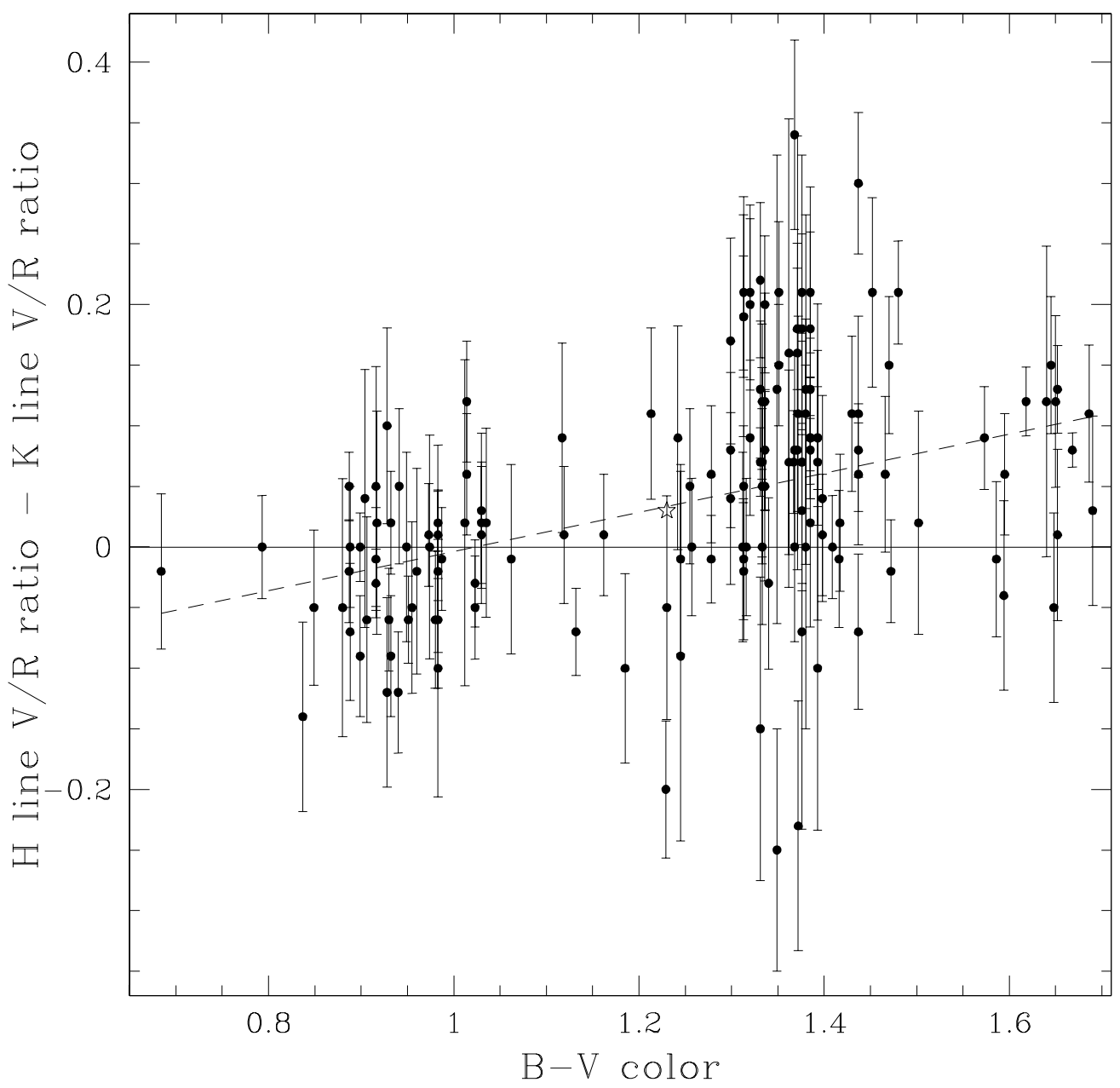

Fig. 6.- Observed values of $(V / R)_{H}-(V / R)_{K}$ versus $B-V$ color. The data are from Table 1 , so some stars show as multiple points in this diagram. The single star symbol represents Arcturus. The dashed line shows a least squares linear fit. 


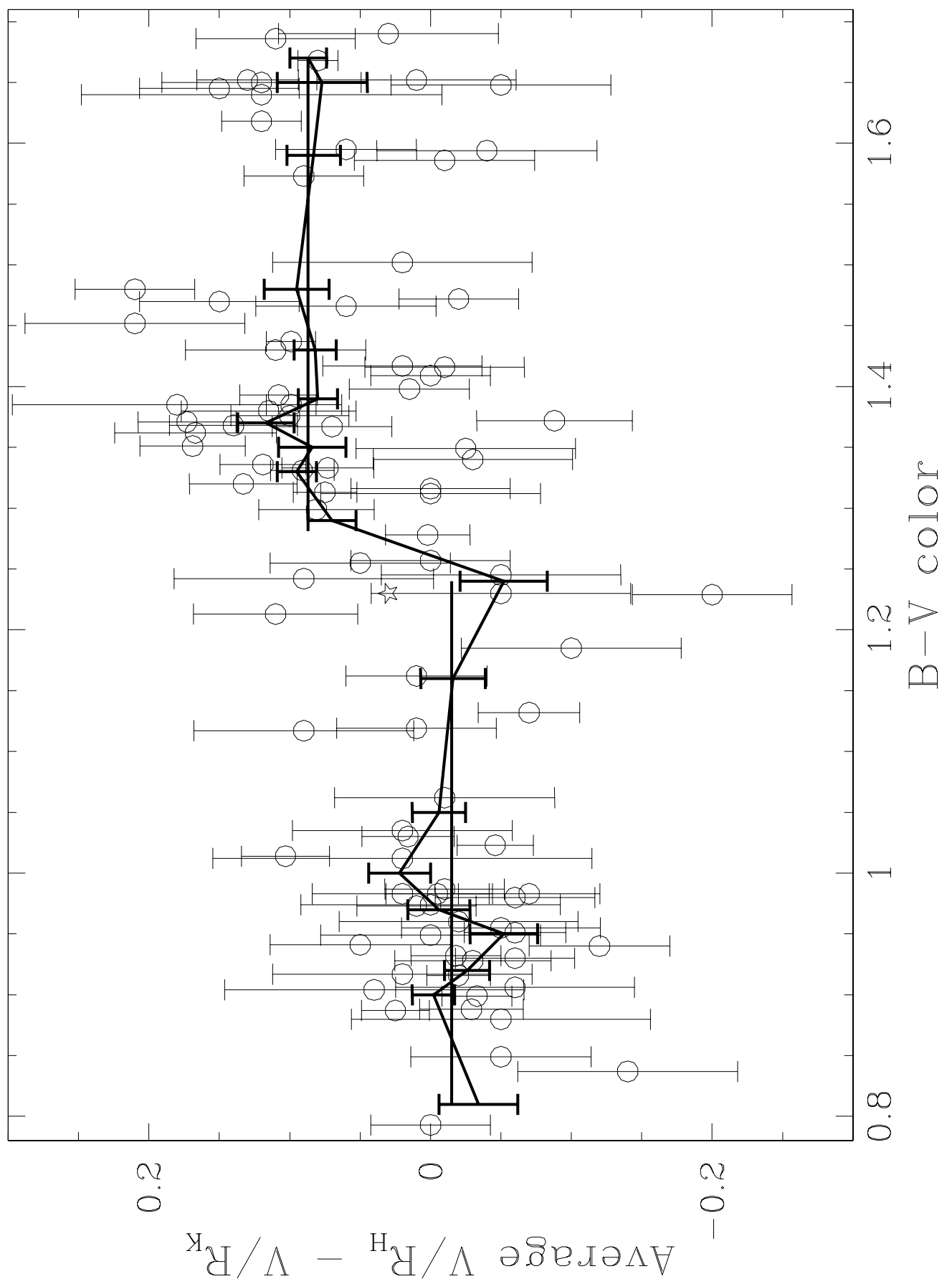

Fig. 7. - The average $(V / R)_{H}-(V / R)_{K}$ value for each star listed in Table 2 versus $B-V$ color. A continuous line connects the weighted mean of every 5 points binned according to $B-V$. This curve shows a rather abrupt change at $B-V \sim 1.25$. The mean values on either side of this "break" are shown by the horizontal lines. 


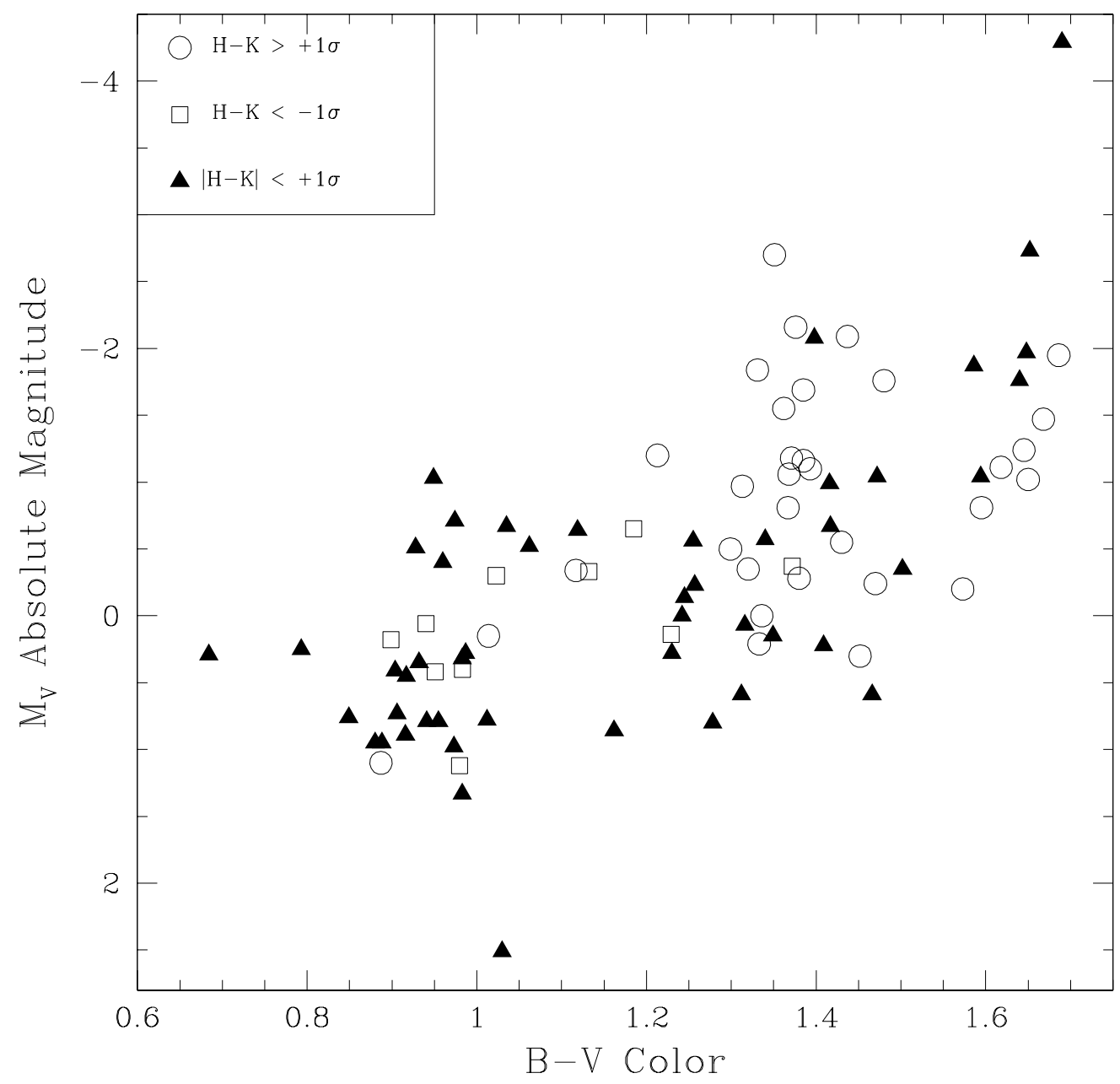

Fig. 8. - The color magnitude diagram of the present sample of giants listed in Table 2. The open circles and open squares represent data points for which the average $\left\langle(V / R)_{H}-\right.$ $\left.(V / R)_{K}\right\rangle>1 \sigma$ or $<-1 \sigma$ respectively. Filled triangles represent data points for which $\left|\left\langle(V / R)_{H}-(V / R)_{K}\right\rangle\right|<1 \sigma$. 


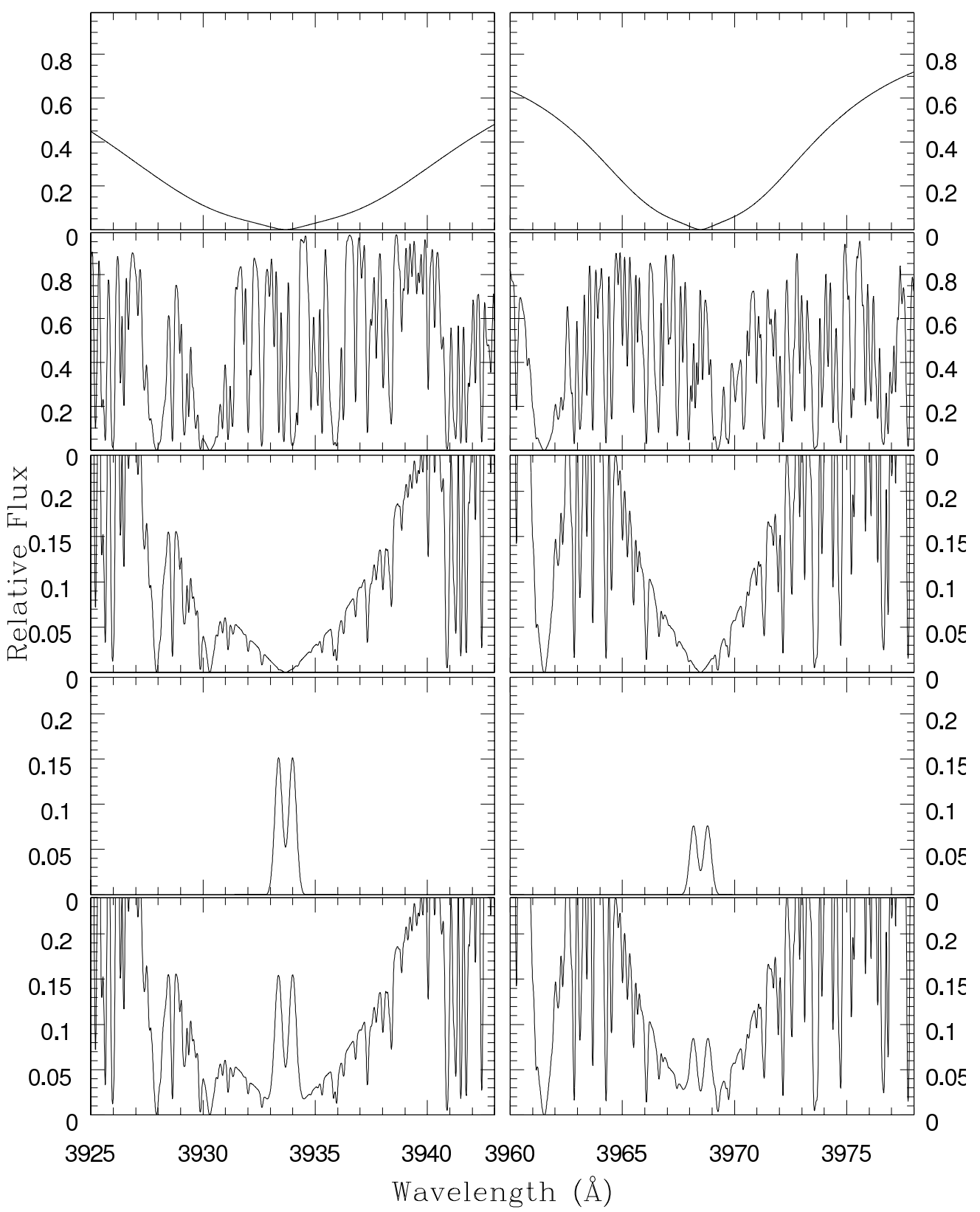

Fig. 9.- Synthetic spectra at wavelengths encompassing the Ca II H and $\mathrm{K}$ lines for a star with $T_{\text {eff }}=4250 \mathrm{~K}, \log g=1.75$, and $[\mathrm{Fe} / \mathrm{H}]=-0.1$. Horizontally paired panels correspond to the regions of the $\mathrm{K}$ (left) and $\mathrm{H}$ (right) lines. Running from top to bottom the panels show: (i) the $\mathrm{H}$ and $\mathrm{K}$ photospheric line profiles alone, (ii) a photospheric spectrum based on a line list that excludes $\mathrm{H}$ and $\mathrm{K}$, (iii) the full photospheric spectrum, (iv) simulated chromospheric emission lines with a ratio of 2:1 in the K:H line strengths, and (v) the summation of the complete synthetic photospheric spectrum plus the simulated emission components. 


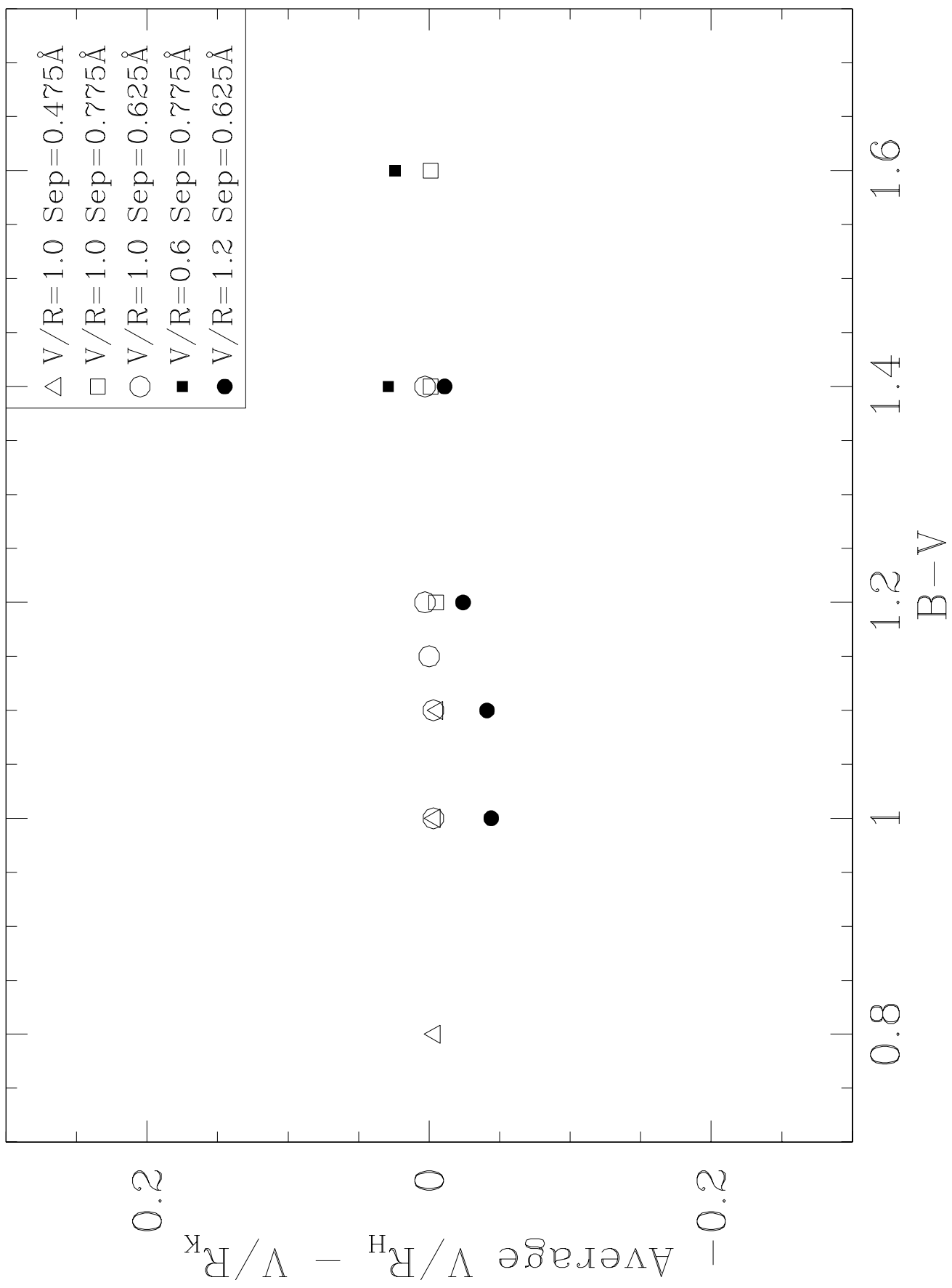

Fig. 10.- The asymmetry difference $(V / R)_{H}-(V / R)_{K}$, found after taking $\mathrm{H}$ and $\mathrm{K}$ emission lines of equal initial $(V / R)$ and superimposing them on a photospheric spectrum, versus the $(B-V)$ color of the photosphere. Symbols denote the morphology of the intrinsic $\mathrm{H}$ and $\mathrm{K}$ emission lines, which are parameterized by their initial $(V / R)$ values and the peak-to-peak separation of the bimodal profiles. 

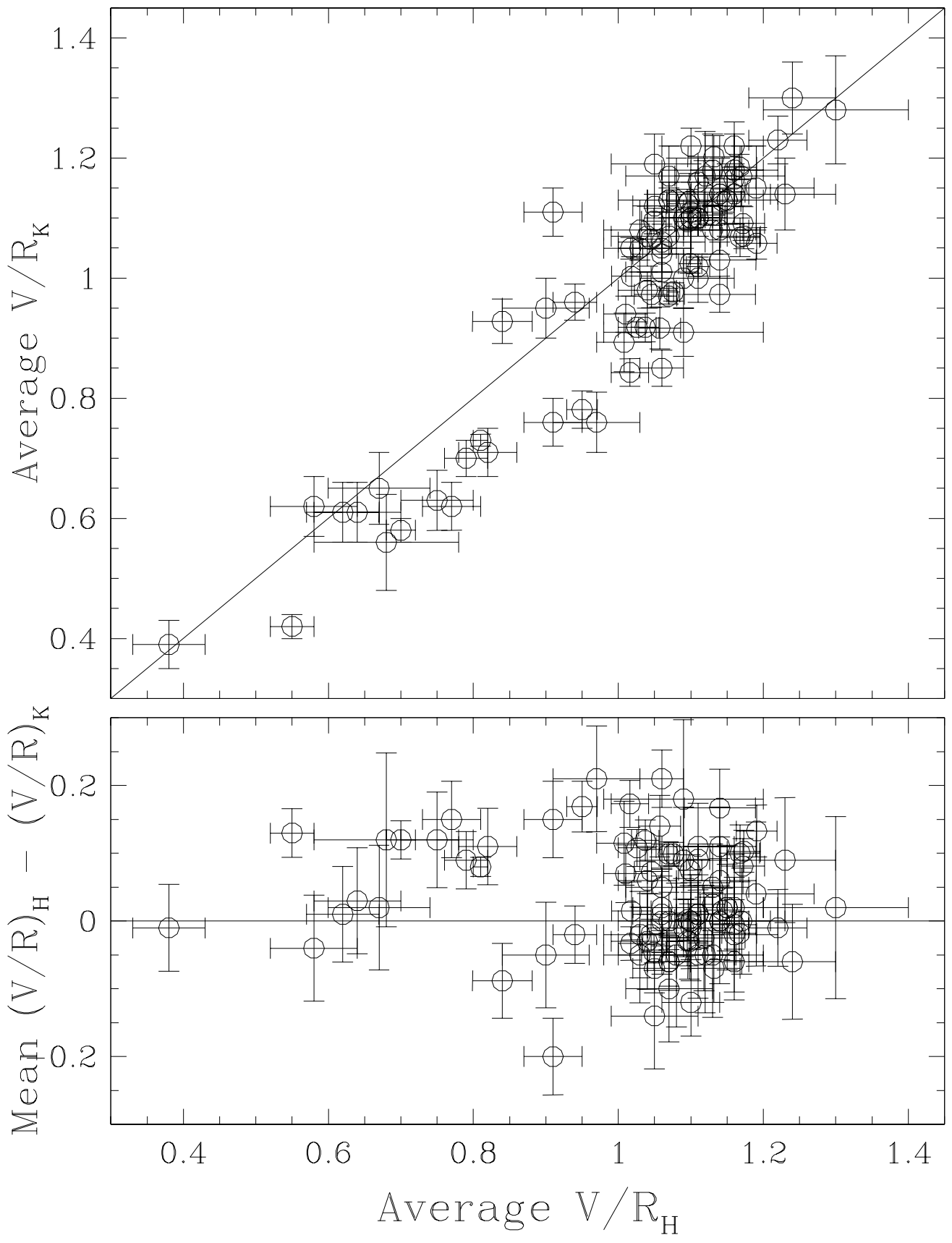

Fig. 11. - The large upper panel shows the average value of $(V / R)_{K}$ versus the average $(V / R)_{H}$ for each star listed in Table 2. The lower panel plots the average value of the asymmetry difference $(V / R)_{H}-(V / R)_{K}$ versus average $(V / R)_{H}$. The solid lines are the locus for $(V / R)_{H}=(V / R)_{K}$. 


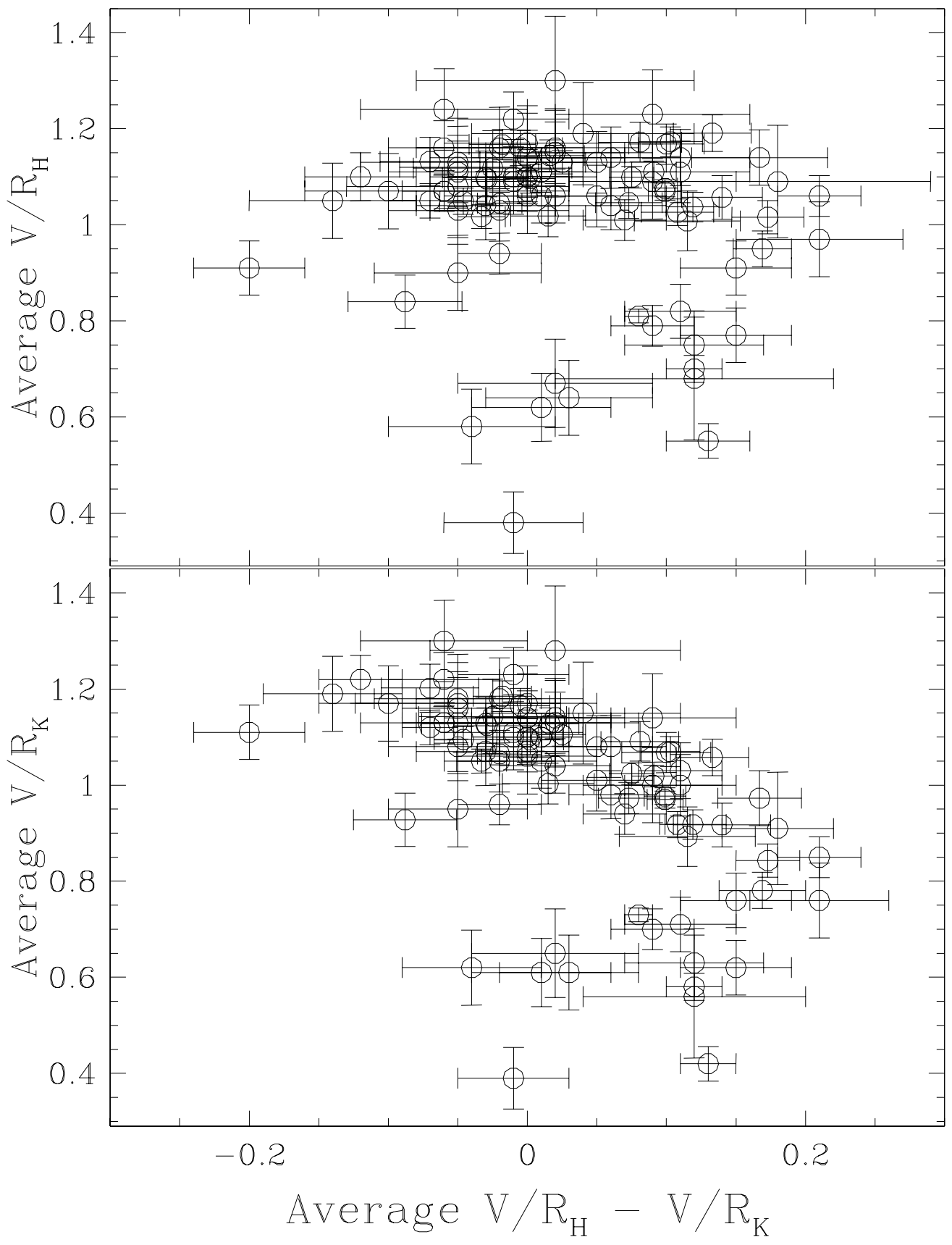

Fig. 12.- The average value of $(V / R)_{H}$ and the average $(V / R)_{K}$ for each star in Table 2 plotted versus the average value of the asymmetry difference $(V / R)_{H}-(V / R)_{K}$. Unlike Figure 5, each star is represented by a single point in this figure. 